\title{
5-Axis Double-Flank CNC Machining of Spiral Bevel Gears via Custom-Shaped Milling Tools - Part II: Physical Validations and Experiments
}

\section{Gaizka Gómez Escudero ( $\square$ gaizka.gomez@ehu.eus )}

Universidad del Pais Vasco Escuela Tecnica Superior de Ingenieria de Bilbao https://orcid.org/00000003-0446-0680

\section{Pengbo Bo}

School of Computer Science and Technology, Harbin Institute of Technology

\section{Haizea González Barrio}

Universidad del Pais Vasco Escuela Tecnica Superior de Ingenieria de Bilbao

\section{Amaia Calleja Ochoa}

Universidad del Pais Vasco Escuela Tecnica Superior de Ingenieria de Bilbao

\section{Michael Barton}

BCAM - Basque Center for Applied Mathematics

\section{Luis Norberto López de Lacalle Marcaide}

Universidad del Pais Vasco Escuela Tecnica Superior de Ingenieria de Bilbao

\section{Research Article}

Keywords: 5-axis CNC machining, double-flank milling, custom-shaped tools, semifinishing operations, tangential movability, free-form shape manufacturing

Posted Date: May 18th, 2021

DOI: https://doi.org/10.21203/rs.3.rs-442857/v1

License: (a) (1) This work is licensed under a Creative Commons Attribution 4.0 International License. Read Full License

Version of Record: A version of this preprint was published at The International Journal of Advanced Manufacturing Technology on November 25th, 2021. See the published version at https://doi.org/10.1007/s00170-021-08166-0. 


\title{
5-axis double-flank CNC machining of spiral bevel gears via custom-shaped milling tools - Part II: physical validations and experiments
}

\author{
Gaizka Gómez Escudero,,a , Pengbo Bo ${ }^{\mathrm{b}}$, Haizea González ${ }^{\mathrm{a}}$, Amaia Calleja ${ }^{\mathrm{a}}$, Michael Bartoñ ${ }^{\mathrm{c}, \mathrm{d}}$, Luis Norberto López de Lacalle \\ ${ }^{a}$ High Performance Manufacturing Group, Department of Mechanical Engineering, \\ the University of the Basque Country, Plaza Ingeniero Torres Quevedo 1, 48013, Bilbao, Basque Country, Spain \\ ${ }^{b}$ School of Computer Science and Technology, Harbin Institute of Technology, West Wenhua Street 2, 264209 Weihai, China \\ ${ }^{c}$ BCAM - Basque Center for Applied Mathematics, Alameda de Mazarredo 14, 48009 Bilbao, Basque Country, Spain \\ ${ }^{d}$ Ikerbasque - Basque Foundation for Sciences, Maria Diaz de Haro 3, 48013 Bilbao, Basque Country, Spain
}

\begin{abstract}
We investigate a recently introduced methodology for 5-axis flank computer numerically controlled (CNC) machining, called double-flank milling [1]. Certain geometries, such as curved teeth of spiral bevel gear, admit this approach where the milling tool has tangential contact with the material block on two sides, yielding a more efficient variant of flank milling. To achieve high machining accuracy, the path-planning algorithm, however, does not look only for the path of the tool, but also for the shape of the tool itself. We validate the approach by series of physical experiments using an abrasive custom-shaped milling tool specifically designed for a particular type of a spiral bevel gear. We show the potential of this new methodology in the semifinishing stage of gear manufacturing, where it outperforms traditional ball end milling by an order of magnitude in terms of machining time, while keeping, or even improving, the machining error.
\end{abstract}

Key words: 5-axis CNC machining, double-flank milling, custom-shaped tools, semifinishing operations, tangential movability, free-form shape manufacturing

\section{Introduction \& Motivation}

Efficient and highly-accurate manufacturing of curved geometries such as car transmissions, gearboxes, or other doublycurved engine parts is a serious challenge in many industries like automotive or aeronautic, to name a few. Spiral bevel gears, when compared to straight-toothed bevel gears, are able to run at higher speed [2] and are therefore indispensable elements among gear mechanisms. To achieve smooth and silent highspeed transmission, manufacturing with a very high precision is essential. Moreover, high precision increases durability of the manufactured gears that is another main objective for modern, sustainable manufacturing technologies [3].

Traditionally, manufacturing of spiral bevel gears requires specially-deviced machines. There are several mainstream approaches to manufacture spiral bevel gears: gear hobbing with perimeter cut (Gleason) [4], cyclo-palloidal continuous generation by spiral hobbing (Klingelnberg and Oerlikon) [5], and continuous generation by spiral hobbing with conical-type cut (Klingelnberg) [2]. However, all these approaches are appropriate for large manufacturing batches.

In contrast, we aim at low-cost manufacturing of a single workpiece and/or replacement of a broken part using 5-axis

\footnotetext{
${ }^{*}$ Corresponding author

Email addresses: gaizka.gomez@ehu .eus (Gaizka Gómez Escudero), pbbo@hit.edu.cn (Pengbo Bo), haizea.gonzalez@ehu.eus (Haizea González), amaia.calleja@ehu.eus (Amaia Calleja), mbarton@bcamath. org (Michael Bartoň), norberto. Izlacalle@ehu. eus (Luis Norberto López de Lacalle)
}

computer numerically controlled (CNC) machining. The recent trends in gear manufacturing already head this direction [6, 7, 8], which is well-suited for small batches and an economical alternative to the traditional approach using an expensive hobbing machine. At the same time, CNC machining is able to keep high-accuracy that is essential for gear manufacturing. Another significant advantage of 5-axis CNC machining comes from the fact that it can be combined with additive manufacturing [9]. This type of hybrid manufacturing enables, for example, gear repair, that is something highly desirable, but not possible with the traditional gear-cutting methods.

The purpose of this study is to further advance our recent geometric modeling simulations on 5-axis CNC machining with custom-shaped milling tools [10]. That is, the path-planning algorithm does not only look for optimal milling paths, but also for the shape of the tool [1, 11, 12]. While flank milling with curved (barrel) tools is known and possible for input freeform surfaces [13, 10] simultaneous tangential contact on two sides requires a specific input geometry. Our numerical simulation results showed that for spiral bevel gears, a custom-shaped tool admits enough freedom to flank-mill a curved valley between two spiral gear teeth with a single sweep, having a bitangential contact throughout the motion. This newly introduced methodology, called double-flank milling, therefore offers even more efficient manufacturing than traditional flank milling. In this paper, we conduct real machining experiments to physically validate the recently proposed methodology and show that this approach outperforms classical ball-end milling 
by order of magnitude in terms of machining time.

\section{Previous work}

Manufacturing of spiral bevel gears has been studied over several past decades, see e.g. [2, 4, 5, 14] and other relevant references in [14]. The whole loop: design, production, inspection and installation of spiral-toothed bevel gears is a complex process that requires a special treatment. There are several geometric constraints that need to be satisfied to guarantee smooth transmission between the cutting tool and the material block: (i) the flank contact between the conjugate gear pair (the tool and tooth) has to be along a whole line (curve), (ii) the line contact is equally distributed in the entire engagement area, and (iii) the line contact needs to preserved at every instant of the motion [4].

Traditional pipeline for manufacturing of gears is aimed for large batches and groove-making machines that rely on slotting on curved tool. The two main approaches: the continuous indexing method, referred to as "face hobbing", and the single indexing method referred to as "face milling" present some differences. The face hobbing method produces an epicycloidal shape in tooth lengthwise direction. The face milling method is processed in such a way that both flanks are manufactured in a single cut, i.e., a constant slot width results in the tooth root due to the circular cutter head [15]. While palloid gears are produced by a conical hob, the cyclo-palloid gears are manufactured using a face mill cutter.

Our paper belongs to a family of modern approaches that focus on gears manufacturing using universal multitasking machines or five-axis milling centers [16, 17]. The main advantage of this new trend stems in its versatility as the tool in general does not depend on the gear geometry. This fact makes the technology very flexible as it can be performed on various milling centers, and not, in contrast to the traditional methods (Gleason and Klingelnberg), on one specific large-scale machine.

Suh et al. [8] use a 3-axis milling machine with a rotary table, however, ball end milling is applied in semi-finishing and finishing stages. A numerical approach for determination of machine-tool settings for roughing of a pinion by using a spread-blade face-milling cutter is proposed in [3]. Five-cut method is applied and the manufacturing time is minimized by maximizing the material cut during the rough-cutting stage. Traditionally, standard tools are frequently used for gears machining in universal machining centers [7]. However, we follow the trend where free-form tools are used for near-to-net-shape machining, especially for complex slots, such as the interdental cavity of the gear.

For certain type of gears, such as non-circular spur bevel gear, the irregular shape makes the design and manufacturing process even more difficult and forging stage is involved to distribute the material according to the shape features of the gear [18]. Another issue that needs to be carefully considered is surface roughness. A model to predict and control the teeth surface roughness for $3+2$ axis milling using ball-end milling has been proposed recently [19].
Other recent works have focused on gears machining with universal machines using milling technology [20, 21]. However, innovative processes with higher material removal rates are one of the main objective in modern manufacturing. In this line, Super Abrasive Machining (SAM) [22] is one versatile and feasible solution that increases gears' machining efficiency. Specifically, SAM provides grinding technology precision [23] with similar machining feeds and costs, but with shorter machining times.

Our research goes in the direction of efficient spiral bevel manufacturing using universal multitasking machines or fiveaxis milling centers, where the main objective is to further reduce machining time. To this end, we aim at the semi-finishing stage using highly efficient double-flank milling where not only the path of the tool, but also the shape of the tool itself are the unknowns in our optimization-based framework. (a)

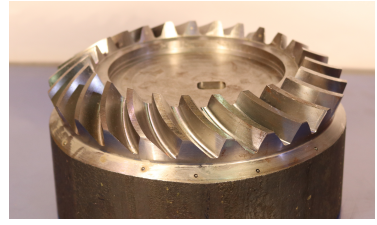

(b)

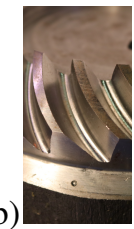

(c)

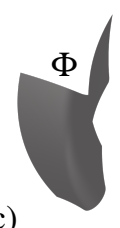

Figure 1: (a) Spiral bevel gear (5-axis CNC machined using our methodology). (b) A zoom-in to one interdental cavity (aka "valley") between two teeth and its CAD model that is formed by a doubly curved free-form surface $\Phi$ (c).

The rest of the paper is organized as follows. Section 3 gives a brief summary of the mathematical derivation of the customshaped tool design and its 3D motion. Section 4 describes the case study and Section 5 shows the results obtained. Finally, Section 6 discusses the future research directions and concludes the paper.

\section{Double-flank milling}

In order to make the paper self-contained, we briefly recall the basic building blocks of the double-flank machining algorithm. We first recall some basics on general milling tools and their rigid body motions (Section 3.1), and then discuss the two main steps of our algorithm: initialization of the tool and its motion to have double-tangential contact (Section 3.2) and global tool and motion optimization (Section 3.3.

\subsection{General milling tools in $3 D$}

Traditionally, conical and cylindrical tools of given slope and size are used for flank milling. In this work, we consider tools that are general (aka custom-shaped) and their shape is a variable in our optimization-based approach. Let $\mathbf{l}:=\mathbf{p q}$ be a finite 3D line, the rotational axis of the milling tool. Then a custom-shaped milling tool $\Psi$ is defined by a scalar function $d(s)$ that assigns to every point of $\mathbf{l}(s)$ a real value. That is, the milling tool is an envelope of a one-parameter family of spheres centered along l, see Fig. (2)(b). The intersection of $\Psi$ and an arbitrary plane that contains $\mathbf{l}$ is known as a meridian.

To control the motion of the milling tool $\Psi$, it is sufficient to control the motion of its rigid axis pq. Let us consider the 


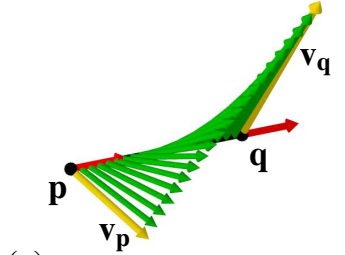

(a)

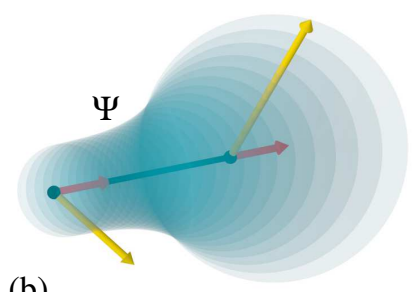

(b)

Figure 2: An instantaneous motion of the axis of the milling tool $\mathbf{p q}$ is determined by two velocity vectors $\mathbf{v}_{\mathbf{p}}$ and $\mathbf{v}_{\mathbf{q}}$ (yellow) that have to satisfy Eq. 22. This constraint is geometrically interpreted as the projections of $\mathbf{v}_{\mathbf{p}}$ and $\mathbf{v}_{\mathbf{q}}$ onto pq are equally oriented vectors of the same length (red). (b) A milling tool $\Psi$ is conceptualized as a one-parameter family of spheres (transparent) centered along pq and determined by $d(s)$; here a hyperbolic tool is shown. (a)

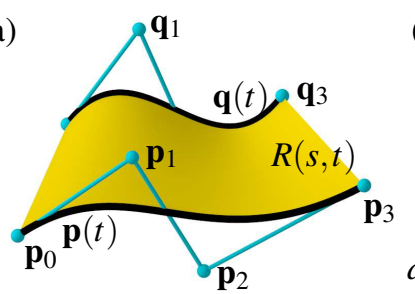

(b)

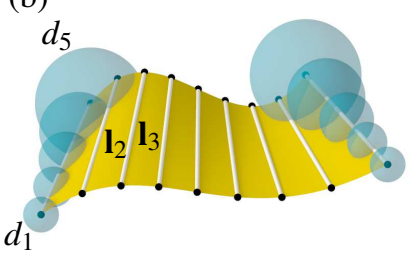

Figure 3: General milling tool and its motion. (a) A motion of the axis $\mathbf{I}$ of the milling tool is a ruled surface $R(s, t)$ that is controlled by two boundary curves $\mathbf{p}(t)$ and $\mathbf{q}(t)$. A single $(1 \times 3)$ tensor-product Bézier patch is shown; the control points $\mathbf{p}_{0}, \ldots, \mathbf{q}_{3}$ (blue) are the unknows for the optimization. (b) The general milling tool is considered as a one-parameter family of spheres centered along $\mathbf{I}$ and represented by a vector of radii $\mathbf{d}=\left(d_{1}, \ldots, d_{n}\right)$ which is also an unknown in our optimization framework (here $n=5, m=9$ ).

trajectories of the endpoints as a function of time, i.e., $\mathbf{p}(t), \mathbf{q}(t)$ $t \in[0,1]$ (for simplicity of the argument we use the unit interval for the time range). To express that the axis is rigid (its length $L$ remains constant during the motion), we write

$$
L^{2}=\|\mathbf{p}-\mathbf{q}\|^{2}=\langle\mathbf{p}-\mathbf{q}, \mathbf{p}-\mathbf{q}\rangle=\text { const. }
$$

where $\langle\cdot, \cdot\rangle$ is the inner product. Differentiating with respect to time $t$ and denoting the velocity vectors by $\mathbf{v}_{\mathbf{p}}=\dot{\mathbf{p}}(t), \mathbf{v}_{\mathbf{q}}=\dot{\mathbf{q}}(t)$, we obtain

$$
\left\langle\mathbf{v}_{\mathbf{p}}, \mathbf{p}-\mathbf{q}\right\rangle=\left\langle\mathbf{v}_{\mathbf{q}}, \mathbf{p}-\mathbf{q}\right\rangle
$$

which is (the first order) length-preserving constraint of $\mathbf{l}$. To control the instantaneous motion of $\mathbf{l}$, one needs to prescribe the two 3D vectors $\mathbf{v}_{\mathbf{p}}$ and $\mathbf{v}_{\mathbf{q}}$ at the endpoints; the remaining velocity are just their linear combinations, see Fig. (2)(a). The two vectors $\mathbf{v}_{\mathbf{p}}$ and $\mathbf{v}_{\mathbf{q}}$ have to satisfy Eq. (2). There are five degrees of freedom for that ( 3 coordinates of $\mathbf{v}_{\mathbf{p}}$ and 2 of $\mathbf{v}_{\mathbf{q}}$ ), see e.g. [24] for more details.

\subsection{Initialization of double flank milling}

We aim to approximate the valley between two teeth of a gear $\Phi$, recall Fig. 1, by an envelope of a general milling tool $\Psi$ such that there is tangential contact between $\Phi$ and $\Psi$ on both sides of the valley (double-flank milling). The unknowns in our optimization-based algorithm are both the milling tool $\Psi$ and a ruled surface $R$ (the motion of the milling axis). The
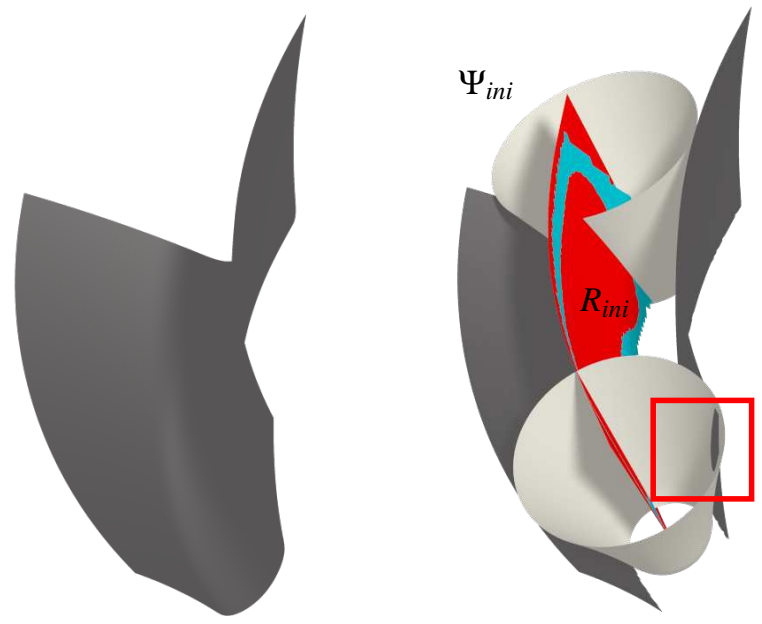

(a)

(b)
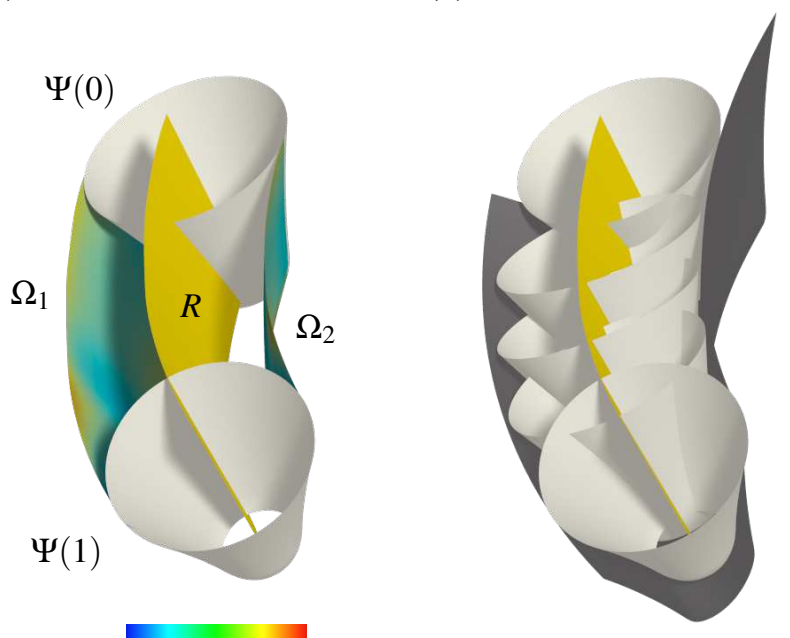

(c) $0 \mu m$

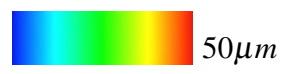

(d)

Figure 4: Design of the custom-shaped tool. (a) A cavity bewteen two teeth represented as a spline surface. (b) The self-bisector $B$ (blue) is fitted by a ruled surface $R_{i n i}$ (red) and an initial shape, $\Psi_{i n i}$, of the tool is computed. The initial tool and its initial position may penetrate the reference surface (framed in red). (c) The tool $\Psi$ and its trajectory $R$ both undergo global optimization to minimize the error of the left $\left(\Omega_{1}\right)$ and right $\left(\Omega_{2}\right)$ envelopes from $\Phi$. The envelopes are color-coded by the distance error $\operatorname{dist}\left(\Phi_{i}-\Omega_{i}\right), i=1,2$, that meets fine machining tolerance of $50 \mu \mathrm{m}$. (d) The final double-flank motion of the custom-shaped tool $\Psi$ through the gear valley.

ruled surface is represented as a $(3 \times 1)$-tensor product B-spline patch

$$
R(t, s)=(1-s) \mathbf{p}(t)+s \mathbf{q}(t), \quad[t, s] \in[0,1] \times[0,1],
$$

where $s$ is the parameter in the direction of rulings and $t$ is the time parameter of the two boundary cubic B-spline curves $\mathbf{p}(t)$ and $\mathbf{q}(t)$, see Fig. 3 .

Given the valley $\Phi$ between two teeth of a gear, we first eliminate the bottom part of the valley (as this part cannot be flank-milled anyway) and define two side surfaces $\Phi_{1}$ and $\Phi_{2}$. As the milling tool is aimed to have tangential contact with both $\Phi_{1}$ and $\Phi_{2}$, we first compute their bisector $B$. The bisector, however, is a general surface, and therefore we use spline fitting method and compute its ruled surface approximation, see 
Fig. 4(b).

The initial ruled surface defines the initial shape of the milling tool $\Psi$ and its envelopes define the initial approximation of the two surfaces $\Phi_{1}$ and $\Phi_{2}$. The two (right and left) envelopes $\Omega_{1}$ and $\Omega_{2}$ are required to approximate $\Phi_{1}$ and $\Phi_{2}$, respectively, as close as possible, and within the given machining accuracy $\varepsilon=50 \mu \mathrm{m}$, see Fig. 4(c).

To compute the best envelopes $\Omega_{1}$ and $\Omega_{2}$, we formulate it as an optimization problem. The unknowns are the two curves $\mathbf{p}(t)$ and $\mathbf{q}(t)$ (boundaries of the rule surface $R$ ) and a scalar function $d(s)$ that determines the sphere radius in the ruling direction $s$. To compute the self-bisector $B$ of $\Phi$, see Fig. 4(b), we conceptualize the motion of the tool as a two parameter family of spheres (one in time, second in the ruling direction) that should ideally touch $\Phi$ on two sides, see Fig. 3 b). $B$ is then a locus of all such centers of spheres. We use the right and left side (defined by trimming off the bottom part of the valley) surfaces $\Phi_{1}$ and $\Phi_{2}$, see Fig. 4(b), to compute $B$, and write

$$
F(\mathbf{z})=\operatorname{dist}\left(\mathbf{z}, \Phi_{1}\right)-\operatorname{dist}\left(\mathbf{z}, \Phi_{2}\right),
$$

where $\mathbf{z} \in \mathbb{R}^{3}$ is the desired center of the sphere and dist is the point-surface minimal distance. We look for the iso-surface $F(\mathbf{z})=0$ that defines our bisector $B$ and use a variant of the marching cubes algorithm, see [1] for more details.

\subsection{Tool and motion optimization}

In our optimization-based framework, we optimize both the milling tool $\Psi$ and its motion, represented by a ruled surface $R$. Our optimization has two main objectives: (i) we want to approximate the surface within a fine machining error, i.e, remove as much material as possible and (ii) guarantee that the envelope of the tool lies inside the valley $\Phi$, i.e., there is no overcut.

As discussed in Section 3.2, we compute an initial ruled surface $R^{i n i}$ from the self-bisector $B$. This gives us also, for each value of $s, s \in[0,1]$, a set of scalar values that correspond to the distance $\operatorname{dist}(R(t, s), \Phi)$ and by averaging these values for various $t$ we obtain an initial radial function $d^{\star}(s)$. This gives an initial pair of envelopes $\Omega_{1}^{i n i}$ and $\Omega_{2}^{i n i}$. However, these envelopes, in general, intersect $\Phi$ which corresponds to overcutting, see Fig. 4(b).

To eliminate this phenomenon, the tool $\Psi_{i n i}$ and its motion $R^{i n i}$ both undergo global optimization. Our aim is to optimize them such that $\Omega_{1}^{i n i}$ and $\Omega_{2}^{i n i}$ become as close as possible to $\Phi$ (remove as much material as possible) and they both lie inside the valley (no overcut). To achieve this goal, we proceed as follows. The ruled surface $R$ is uniformly sampled both in $t$ and $s$ parametric directions to obtain $\mathbf{r}_{i j}:=R\left(t_{i}, s_{j}\right), i=1, \ldots, m, j=$ $1, \ldots, n$. In our discrete approach, for each $s$-parameter value (fixed $j$ ), we obtain a set of discrete values $d_{i j}$ which are the distances from $\Phi$ for a fixed point of the axis as it moves in time. To obtain a motion of $\Psi$ that is penetration-free with $\Phi$ (no overcut), we define

$$
\underline{d}_{j}=\min _{i=1, \ldots, m} d_{i j}
$$

where $\underline{d}_{j}$ are the penetration-free radii. In this discrete setup, the penetration-free radius depends on the sampling density. We use $m=100$ in our experiments that turned out to be sufficiently large to return stable values for the valley shown in Fig. 1 .

These radii are the lower bounds of the point-surface distance for each $j$, i.e., distances that define (discrete) radial function that corresponds to a penetration-free tool, see Fig. 4(d). We further define the penetration-free error as

$$
\varepsilon_{j}=d_{j}^{\star}-\underline{d}_{j}
$$

where $d_{j}^{\star}$ are the samples of the initial radial function $d^{\star}$. We denote by $\mathbf{d}$ a vector of unknown distances $\mathbf{d}:=\left(d_{1}, \ldots, d_{n}\right)$ and optimize both, the ruled surface $R$ and d, see Fig. 3. (c).

Finally, we formulate our objective that, at every time instant $t$, the tool is required to be as close as possible to $\Phi$, but also penetration-free. This leads to a minimization problem

$$
F_{\text {prox }}(\mathbf{p}, \mathbf{q}, \mathbf{d})=\frac{1}{m n} \sum_{j=1}^{n} \sum_{i=1}^{m}\left(\operatorname{dist}\left(\mathbf{r}_{i j}, \Phi\right)-d_{j}-\varepsilon_{j}\right)^{2} \rightarrow \min
$$

subject to the axis-rigidity constraints

$$
F_{\text {rigid }}(\mathbf{p}, \mathbf{q})=\left\langle\mathbf{p}\left(t_{i}\right)-\mathbf{q}\left(t_{i}\right), \mathbf{p}\left(t_{i}\right)-\mathbf{q}\left(t_{i}\right)\right\rangle-L^{2}=0,
$$

where $\operatorname{dist}($,$) is a point-surface distance and L$ is the length of $\mathbf{l}$. The unknowns in the minimization are the control points of the two B-spline curves $\mathbf{p}(t)$ and $\mathbf{q}(t)$, and the vector of sphere radii d. We set $m=100$ and $n=30$ for our numerical simulations.

\section{Case study}

The path-planning algorithm described above was implemented into a G-code and tested in a conventional machining center, Kondia HS1000, see Fig 5 The semifinishing operations were carried out with both a ball-end tool and a customshaped tool. The custom-shaped tool was capable of bitangential machining, which resutled in a simultaneous semifinishing of both walls of the interdental cavity. One of the objective was to reduce machining times in the semifinishing stage as the custom-shaped tool admits wide strips of high accuracy and therefore only a single path is needed, in contrast to ball-end milling which requires many milling paths.

Spiral bevel gear was selected as a case study as it is one of the most widespread components in the industrial sector. It is also the element par excellence used to transfer power from one element to another, by transmitting circular motion in terms of the gear wheel contact. One of the most important applications of gears is the transmission of movement from the shaft of a power source, such as an internal combustion engine or an electric motor, to another, end-effector, shaft. In either case, a high accuracy is highly demanded as the machining errors influence significantly the performance.

The gear wheels can be manufactured from a wide variety of materials to obtain the right mechanical properties. From the point of view of mechanical design, strength and durability, i.e., wear resistance, are the most important attributes. In 


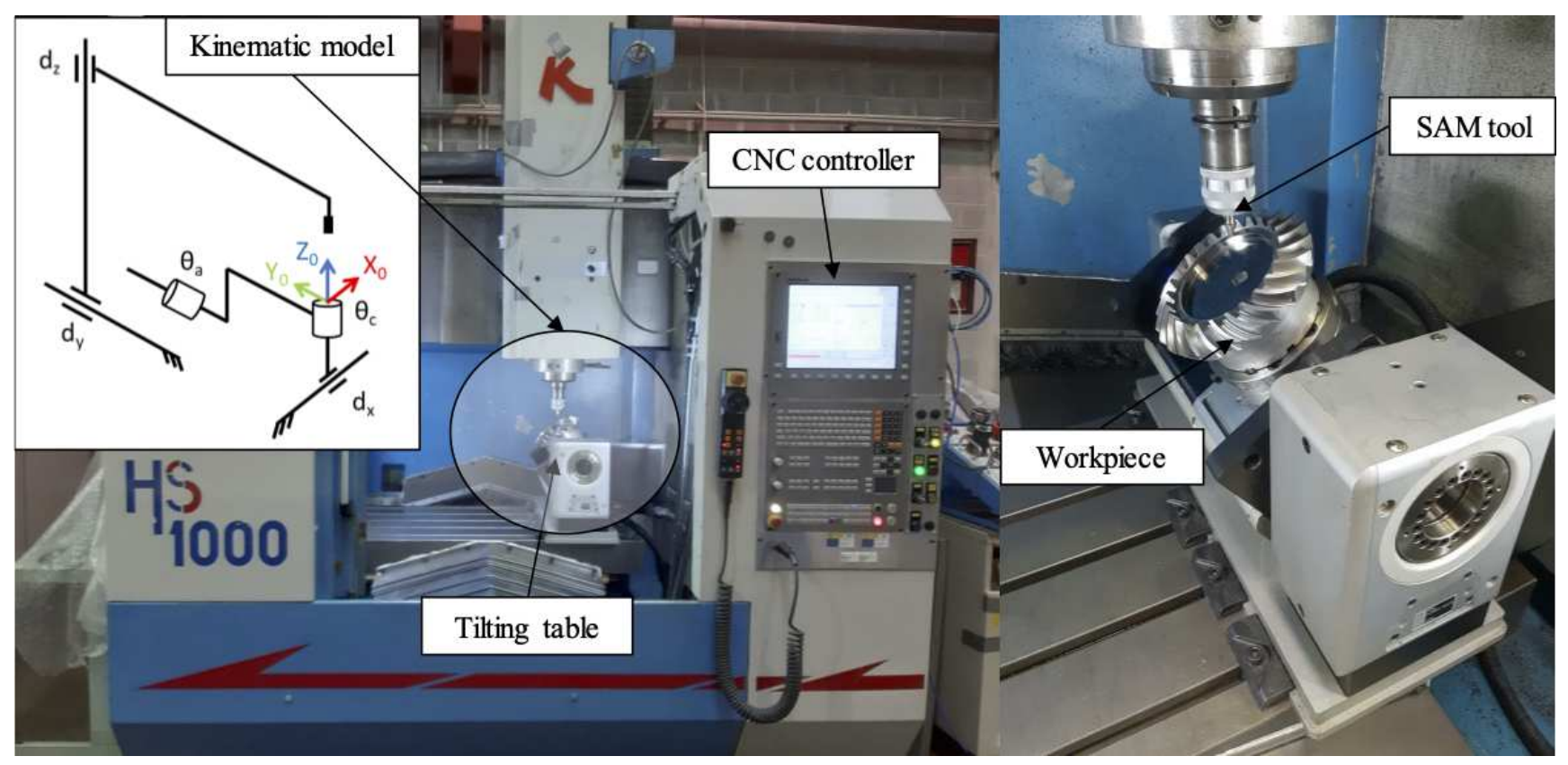

Figure 5: 5-axis milling center.

Table 1: Top: Inconel 718 chemical composition (\%). Bottom: Mechanical and physical properties.

\begin{tabular}{cccccccc}
\hline $\mathbf{C}$ & $\mathbf{S i}$ & $\mathbf{M n}$ & $\mathbf{P}_{\max }$ & $\mathbf{S}_{\max }$ & $\mathbf{C r}$ & $\mathbf{M o}$ & $\mathbf{C u}_{\max }$ \\
\hline $0.15-0.21$ & $0.15-0.40$ & $0.60-0.90$ & 0.025 & 0.035 & $0.90-1.20$ & $0.15-0.25$ & 0.40 \\
\hline Hardness & Yield point & Tensile Strength & \multicolumn{2}{c}{ Density } \\
\hline \multicolumn{2}{c}{$34 \mathrm{HRC}$} & \multicolumn{2}{c}{$0.88 \mathrm{GPa}$} & \multicolumn{2}{c}{$1.08 \mathrm{GPa}$} & $7850 \mathrm{~kg} / \mathrm{m}^{3}$ \\
\hline
\end{tabular}

Table 2: Parameters of a spiral bevel gear.

\begin{tabular}{ll}
\hline External diameter $(\mathrm{De})$ & $207.6 \mathrm{~mm}$ \\
Heel pitch diameter $(\mathrm{Dp})$ & $200 \mathrm{~mm}$ \\
Number of teeth $(\mathrm{Z})$ & 25 \\
Spiral angle $(\beta)$ & $35^{\circ}$ \\
Face angle & $59.5^{\circ}$ \\
Pressure angle $(\alpha)$ & $20^{\circ}$ \\
\hline
\end{tabular}

general, the gear designer should consider the ability to manufacture the gear, from the formation of the gear teeth up to the final assembly of the gear in a machine. Other considerations include weight, corrosion resistance, noise, and cost. F-1550 steel (18CrMo4) was selected as our testing material for manufacturing of the spiral bevel gear, since it reaches a fairly good agreement with all the characteristics that steel needs to possess. Mechanical and physical properties and chemical composition of the used materials are shown in Table 1 .

This particular spiral bevel was chosen, because its contact surface is larger compared to those of straight-toothed bevel gears, and this fact poses a great challenge when computing both the tool geometry and the machining path. The specific characteristics of the gear are shown in Table 2 .

The tests were carried out on a conventional machining center, Kondia HS1000. This machine is a 5-axis milling machine, with 3 linear and 2 are rotary axes. The linear axes are 2 in the head $(\mathrm{X}, \mathrm{Z})$ and one in the table $(\mathrm{Y})$, while both rotary axes are in the indexing table $(\mathrm{A}, \mathrm{C})$. The spindle speed capacity is $24,000 \mathrm{rpm}$.

Initially a $210 \mathrm{~mm}$ diameter and $120 \mathrm{~mm}$ thick steel billet was used as a starting point. A series of previous operations were carried out to achieve a geometry close to the final. These operations are shown in Fig. 6 together with machining times needed for each particular machining stage.

\subsection{Custom-shaped tool for SAM}

Regarding the SAM tool, a custom-shaped tool was used to perform the semifinishing operation of the interdental cavity. Its coat was a monolayer electroplated $\mathrm{CBN}$ grinding with a grain size of $300 \mu \mathrm{m}$, see Figure 7. A tool of this type was chosen due to its excellent tool wear characteristics, because the abrasive grains are resharpened as they break up during the machining process and also because the SAM process is well adapted to the calculation and manufacturing of a custom-shaped tool. When manufacturing the tool core, the thickness of the binding material and the abrasive grains were taken into account in order to obtain a tool with the exact geometry calculated in the modeling stage. The radius of the tool varies from $4.8 \mathrm{~mm}$ to $13.2 \mathrm{~mm}$, see Figure 8 , and the thickness of the abrasive coat is $0.5 \mathrm{~mm}$. Note that the tool has negative Gaussian curvature (i.e., it is not a conical tool), see [1] for more detailed analysis on the tool design. 


\begin{tabular}{|c|c|c|c|}
\hline $\begin{array}{c}\begin{array}{c}\text { Manufacturing } \\
\text { operations }\end{array} \\
\end{array}$ & $\begin{array}{c}\text { Machining } \\
\text { time }\end{array}$ & CAM trajectories & Progress status \\
\hline 0. Blank & - & - & \\
\hline $\begin{array}{l}\text { 1. Preparation of general } \\
\text { gear geometry }\end{array}$ & 2:19:00 & & \\
\hline 2. Refence pocket milling & $0: 01: 14$ & & \\
\hline 3. Roughing gear teeth & 2:31:18 & & \\
\hline 4. Semifinishing gear teeth & - & & - \\
\hline Option A & & & \\
\hline $\begin{array}{l}\text { Ball-end milling } \\
\text { (conventional) }\end{array}$ & $0: 52: 55$ & & \\
\hline Option B & & & \\
\hline SAM (novelty) & $0: 10: 00$ & & \\
\hline 5. Finishing gear teeth & $3: 07: 30$ & & \\
\hline
\end{tabular}

Figure 6: Progress and machining time summary of the whole gear manufacturing process.
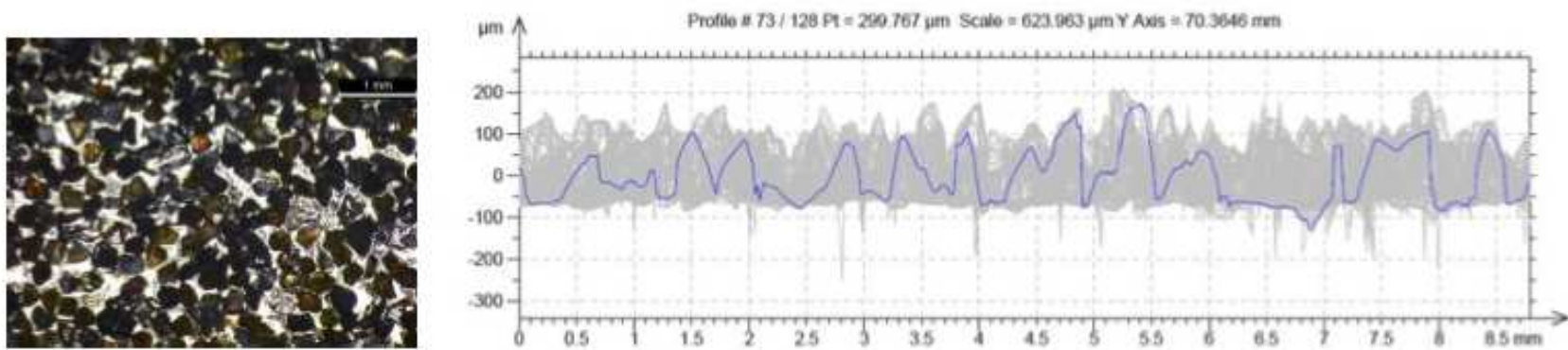

Figure 7: Grain distribution and profile.

Concerning cutting conditions, these were adapted towards the SAM technology in this sort of machining centers, in particular they were adjusted to the spindle capacities, with a spindle speed of 24,000 rpm, the limit of the machine, and a feed rate of $250 \mathrm{~mm} / \mathrm{min}$. It is important to note the effect of cutting speed on machining, especially in the context of super abrasive ma- 

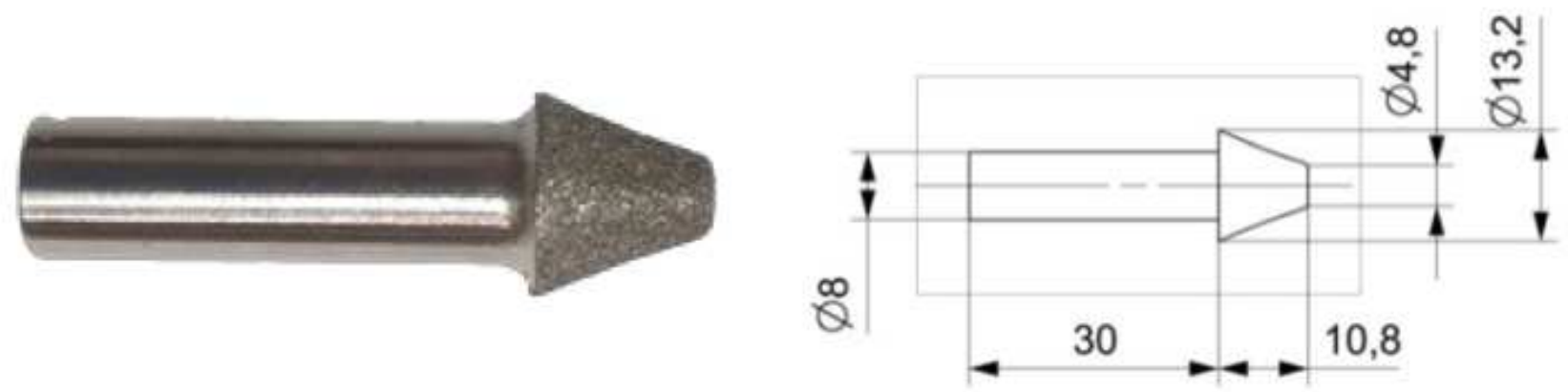

Figure 8: Custom-shaped grinding tool and its geometry.

chining. The optimal SAM conditions of the speed of rotation should be around 60,000-90,000 revolutions [25]. However, to achieve these speeds, high performance heads are required which conventional machines do not have.

\section{Results and validation}

To physically validate the results of our modeling algorithm, we applied the machining path of the custom-shaped tool in the manufacturing of a spiral bevel gear. More specifically, the custom-shaped tool was used in the semifinishing operation, which is the place where Super Abrasive Machining technology has its potential niche of work. In Figure 9 the valley between spiral bevel gear teeth is shown during roughing and semifinishing operations with milling and SAM operations. Observe a clearly visible difference in the quality of surface smoothness in Figure 9 a) and (c).

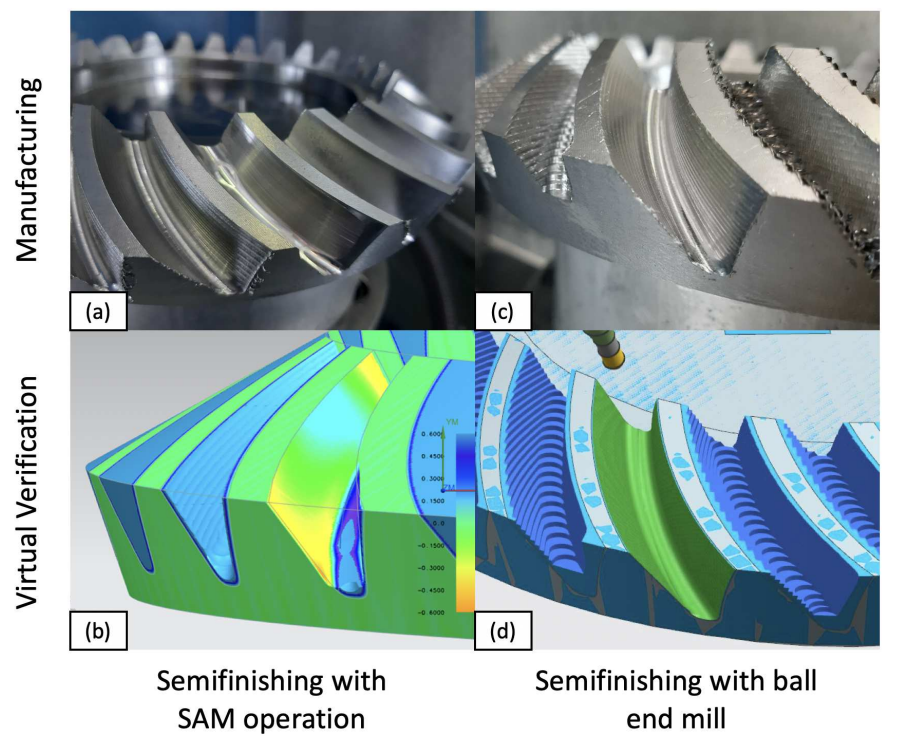

Figure 9: Spiral bevel semifinishing operation. (a) Manufacturing with SAM. (b) Virtual verification with SAM. (c) Manufacturing with ball end mill. (d) Virtual verification with ball end mill.

We further present a qualitative comparison between doubleflank milling using the custom-shaped tool and ball end milling during the semifinishing stage. In particular, we show that surface roughness and manufacturing time are significantly reduced.

\subsection{Surface Roughness}

Surface roughness is one of the key parameters that influence a smooth movement between gears, their face-face contact, and consequently the life of the whole gear. Typically, the surface roughness is measured using a confocal microscope, however, due to the difficult accessibility of the faces of the gear, we applied resin in order to measure a negative of the interdental cavity.

The process for obtaining the negative of the face proceeds as follows: first, the area to be measured is degreased with the DN1 degreaser cleaner provided by PLASTIFORM's own company (PLASTIFORM, Madrid, Spain). Once this is done, a closed area must be formed such that the fluid (liquid resin) covers both sides of the cavity, and then the fluid is applied to the measuring area using a dispensing gun. Finally, cca 6 minutes is needed for the solution to dry out, and then one can remove the negative of the cavity, see Fig. 10

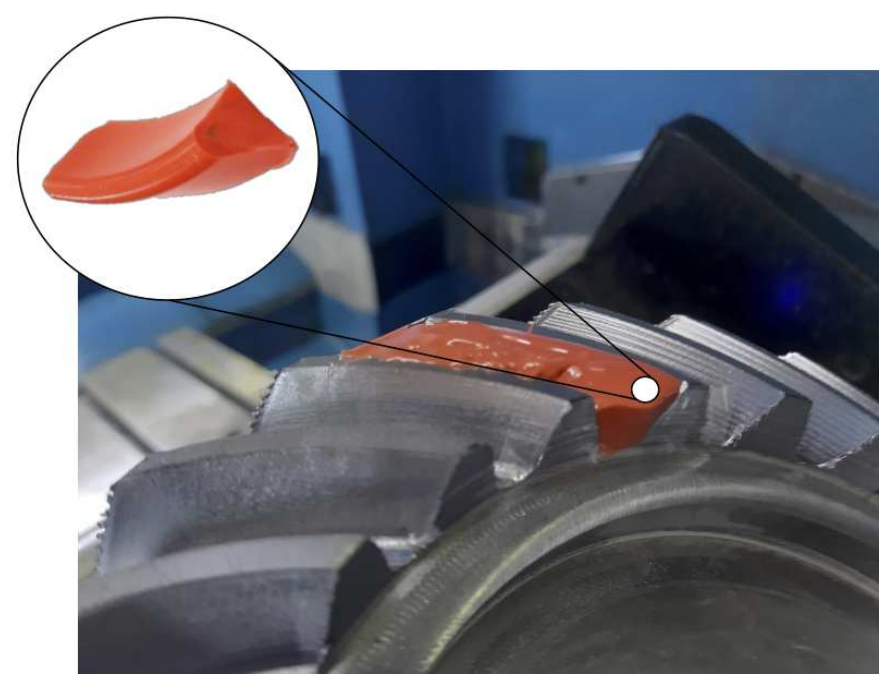

Figure 10: Curing process of the resin and its zoomed-in part after hardening.

It was selected a fluid type resin, so that it could flow through the entire gear cavity and thus adapt well to the surface of the 
faces. Specifically, the F65 product was used, which allows a semi-flexible geometry to be obtained, suitable for measurement by both contact measuring systems and optical measurement systems. The precision obtained with this resin is $\pm 1 \mu \mathrm{m}$.

A Leica DCM 3D confocal microscope was used to analyze the surface roughness of the resin. Both sides of the cavity were analyzed, as the amount of excess of material was slightly different on each side after roughing. The adjustment of the roughness measurement in this case was a cutting length of 0.8 $\mathrm{mm}$ and an evaluation length of $4 \mathrm{~mm}$, according to ISO 4288 [26]. Figure 11 shows the topography and related data of both sides of the cavity.

Topography on both sides of the tooth cavity shows a perfectly recognizable grinding pattern, in which there are remarked peaks and valleys along the scanned surface caused by the random distribution of the abrasive grains. It is noticeable that slightly better results in term of roughness were obtained on the right face, as the roughing operation leaves that face smoother and stepless between passes, just the opposite of the left face, as it can be seen in Figure 9 d). However, this fact is not a limitation of the proposed double-flank aproach, but it is due to the fact that the roughing stage left the right face smoother.

The resutls are in accordance with "indicative surface roughness comparison" that many companies handle [27]. The roughness values obtained were acceptable for a semifinishing operation because they are close to those obtained with similar strategies considering them as finishing operations.

\subsection{Execution Time}

Another aspect that was considered in this work was the analysis of machining time during semifinishing operations on gear teeth. The conventional semifinishing operation using a ball end mill with $4 \mathrm{~mm}$ diameter was used with a stepover of $0.33 \mathrm{~mm}$ of depth of cut in 3 lateral steps in each face and a feed of $2800 \mathrm{~mm} / \mathrm{min}$. On the left face, 20 axial passes were repeated 3 times axially ( 60 passes total) while on the right face 20 passes were sufficient. In contrast, semifinishing using a custom-shaped tool was accomplished in a single sweep with the following parameters: a feed of $500 \mathrm{~mm} / \mathrm{min}$ and a spindle speed of $16000 \mathrm{rpm}$.

With the above-mentioned values of cutting parameters for both manufacturing semifinishing operations, the following machining time results were obtained: (i) conventional ball milling: 2 minutes and 7 seconds and (ii) SAM semifinishing with the custom-shaped tool: 24 seconds. As a result, the semifinishing machining time was reduced by $81.1 \%$, saving in total 43 minutes per gear. See Figure 6 for the summary of the machining times of each particular stage.

\subsection{Discussion and Limitations}

Our approach significantly reduces the semifinishing time by using a properly designed custom-shaped tool. The tool has to be manufactured in advance, however, the custom-shaped tool costs are low, in particular: cylindrical steel bar F115 (85€) to create 4 SAM tools, i.e. $21.25 €$ per the steel core of the tool, and $45 €$ to add the abrasive grains. In total, the cost of the custom-shaped tool is $66.25 €$. In contrast, the on-market tool for ball end milling, VF4SVBR0200, costs $120 €$.

The surface roughness values range $\mathrm{Sa}=2.59-3.87 \mathrm{~mm}$ using the SAM approach which is a slightly more than the numbers that can be obtained by means of conventional milling [27]. Nevertheless, these values are acceptable in the case of a workpiece which later undergoes finishing operations.

Another slight limitation is that the very bottom of the cavity is not accessible with the tool whose shape is designed to double-flank the two faces of the cavity. The bottom of the cavity has to be machined using ball end milling approach.

\section{Conclusion and Future Work}

We have presented a new variant of 5-axis flank milling, called double-flank milling. In this machining methodology, not only the milling path, but also the shape of the tool itself are the unknowns in an optimization-based framework. We have validated the numerical simulations received in [1] by physical manufacturing of a steel spiral bevel gear. A custom-shaped SAM tool was designed and created for this purpose and applied in the semifinishing stage. The physical results confirmed the results obtained in the simulation stage, namely that the machining error gets reduced, the surface roughness is within a standard range, and most remarkably, the machining time of the semifishing stage is reduced by order of magnitude. These are very promising results towards future development of doubleflank milling as a standard manufacturing technology.

As a future research we aim to further develop this methodology and focus on other geometries, e.g., blades and screw rotors, that seem to be well-suited for double-flank milling methodology using custom-shaped tools.

Acknowledgments. The second author has been partially supported by the National Natural Science Foundation of China (Grant No. 61672187 and No. 62072139). The fifths author has been partially supported by Spanish Ministry of Science, Innovation and Universities: Ramón y Cajal with reference RYC-2017-22649 and PID2019-104488RB-I00. The remaining authors were supported by the European Union's Horizon 2020 research and innovation programme under agreement No. 862025 and by the Elkartek funding program, grant no. KK-2020/00102.

\section{Declarations.}

- Funding - See above the Acknowledgments.

- Conflict of interest - The authors hereby confirm that there is no conflict of interest related to this manuscript.

- Data availability - The data (the shape of the tool + its motion), as a part of European Union's Horizon 2020 project, will be available on the project homepage.

- Code availability - The source may undergo other IP issues (e.g. patenting) and will therefore not be publicly available. 

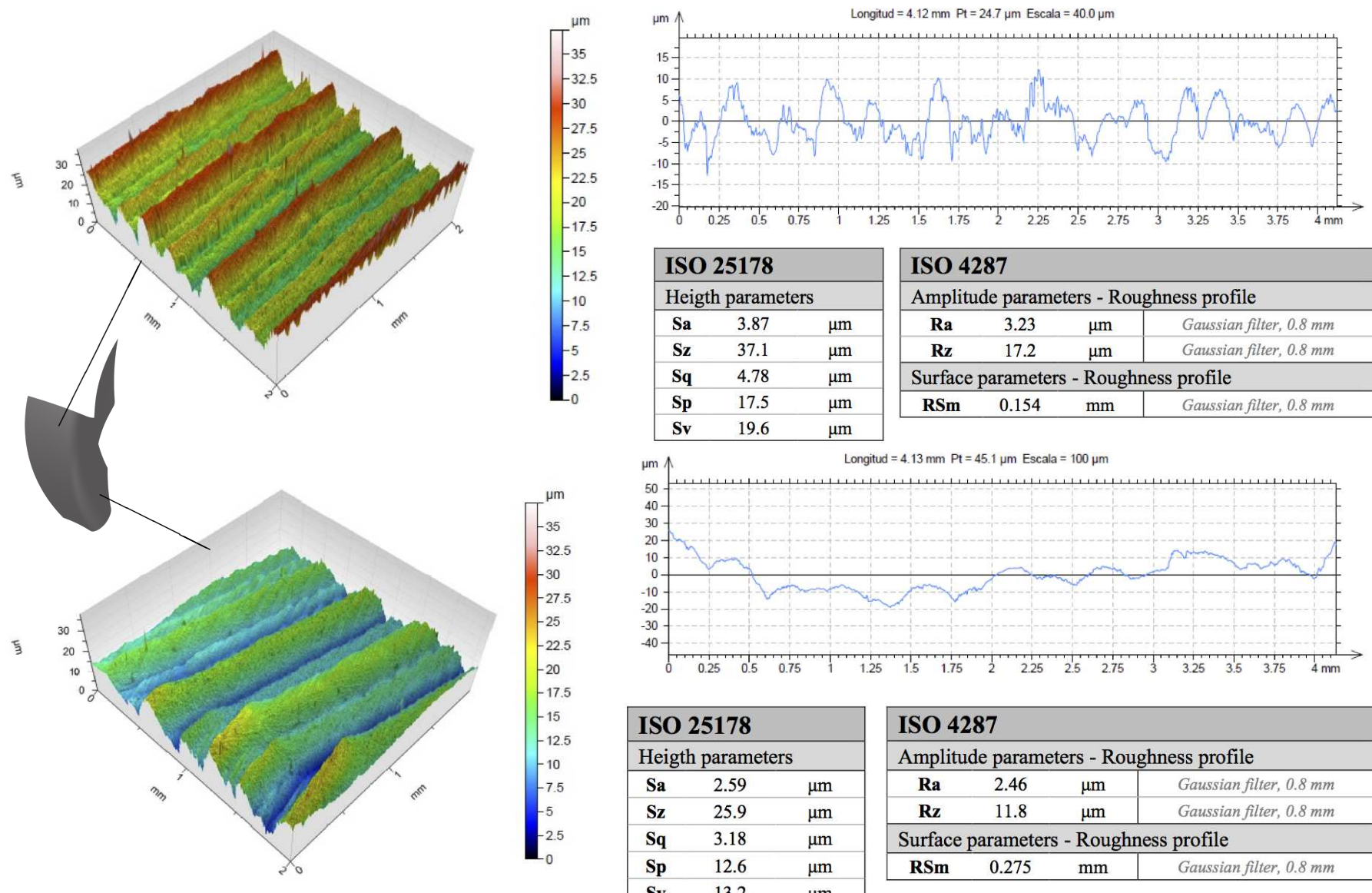

\begin{tabular}{|lll|}
\hline \multicolumn{3}{|l|}{ ISO 25178 } \\
\hline \multicolumn{3}{|l|}{ Heigth parameters } \\
\hline Sa & 3.87 & $\mu \mathrm{m}$ \\
\hline Sz & 37.1 & $\mu \mathrm{m}$ \\
\hline Sq & 4.78 & $\mu \mathrm{m}$ \\
\hline Sp & 17.5 & $\mu \mathrm{m}$ \\
\hline Sv & 19.6 & $\mu \mathrm{m}$ \\
\hline
\end{tabular}

\begin{tabular}{|l}
\hline \multicolumn{1}{|l|}{ ISO 4287} \\
\hline \begin{tabular}{|ccc|c|}
\hline Amplitude parameters - Roughness profile \\
\hline Ra & 3.23 & $\mu \mathrm{m}$ & Gaussian filter, $0.8 \mathrm{~mm}$ \\
\hline $\mathbf{R z}$ & 17.2 & $\mu \mathrm{m}$ & Gaussian filter, $0.8 \mathrm{~mm}$ \\
\hline Surface & parameters & - Roughness profile \\
\hline RSm & 0.154 & $\mathrm{~mm}$ & Gaussian filter, $0.8 \mathrm{~mm}$ \\
\hline
\end{tabular}
\end{tabular}

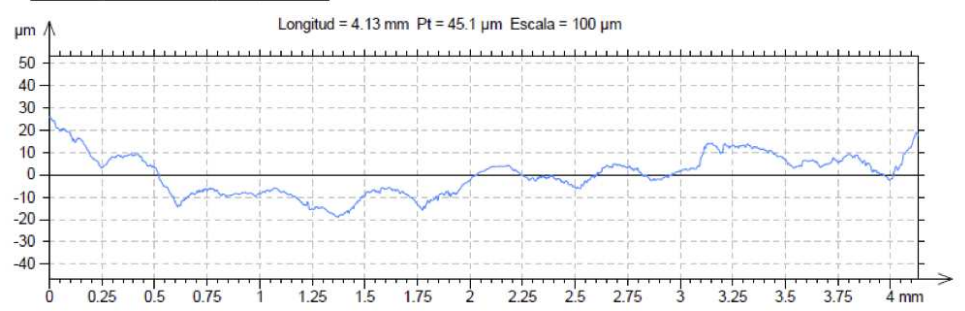

\begin{tabular}{|ccc|}
\hline \multicolumn{3}{|l|}{ ISO 25178} \\
\hline \multicolumn{3}{|l|}{ Heigth parameters } \\
\hline Sa & 2.59 & $\mu \mathrm{m}$ \\
\hline Sz & 25.9 & $\mu \mathrm{m}$ \\
\hline Sq & 3.18 & $\mu \mathrm{m}$ \\
\hline Sp & 12.6 & $\mu \mathrm{m}$ \\
\hline Sv & 13.2 & $\mu \mathrm{m}$ \\
\hline
\end{tabular}

\begin{tabular}{|c|c|c|c|}
\hline \multicolumn{4}{|c|}{ ISO 4287} \\
\hline \multicolumn{4}{|c|}{ Amplitude parameters - Roughness profile } \\
\hline $\mathbf{R a}$ & 2.46 & $\mu \mathrm{m}$ & Gaussian filter, $0.8 \mathrm{~mm}$ \\
\hline $\mathbf{R z}$ & 11.8 & $\mu \mathrm{m}$ & Gaussian filter, $0.8 \mathrm{~mm}$ \\
\hline \multicolumn{4}{|c|}{ Surface parameters - Roughness profile } \\
\hline RSm & 0.275 & $\mathrm{~mm}$ & Gaussian filter, $0.8 \mathrm{~mm}$ \\
\hline
\end{tabular}

Figure 11: Surface area and profile roughness parameters values of the left (top) and right (bottom) side of the gear tooth cavity.

- Ethics approval - The authors confirm that there are no ethical issues related to this paper.

- Consent to participate - All the authors are aware of being co-authors and gave their consents.

- Consent for publication - If accepted, the authors give their consent to the journal to publish and further distribute the article according to the journal policies.

\section{References}

[1] Pengbo Bo, Haizea González, Amaia Calleja, Luis Norberto López de Lacalle, and Michael Bartoň. 5-axis double-flank CNC machining of spiral bevel gears via custom-shaped milling toolspart I: Modeling and simulation. Precision Engineering, 62:204-212, 2020.

[2] W. Krumme. Klingelnberg-Spiralkegdräder (Klingelnberg Spiral Bevel Gears) (in German). Springer, 1967.

[3] Alfonso Fuentes-Aznar, Ramon Ruiz-Orzaez, and Ignacio GonzalezPerez. Numerical approach for determination of rough-cutting machinetool settings for fixed-setting face-milled spiral bevel gears. Mechanism and Machine Theory, 112:22-42, 2017.

[4] Hermann J Stadtfeld. Gleason Bevel Gear Technology: The Science of Gear Engineering and Modern Manufacturing Methods for Angular Transmissions. Gleason Works, 2014.

[5] Henry John Watson. Modern gear production. Elsevier, 2013.

[6] Xiao-zhong Deng, Geng-geng Li, Bing-yang Wei, and Jing Deng. Facemilling spiral bevel gear tooth surfaces by application of 5-axis CNC machine tool. The International Journal of Advanced Manufacturing Technology, 71(5-8):1049-1057, 2014.
[7] Álvaro Álvarez, Amaia Calleja, Naiara Ortega, and Luis de Lacalle. Fiveaxis milling of large spiral bevel gears: toolpath definition, finishing, and shape errors. Metals, 8(5):353, 2018.

[8] SH Suh, WS Jih, HD Hong, and DH Chung. Sculptured surface machining of spiral bevel gears with CNC milling. International Journal of Machine Tools and Manufacture, 41(6):833-850, 2001.

[9] Ian Gibson, David W Rosen, Brent Stucker, et al. Additive manufacturing technologies, volume 17. Springer, 2014.

[10] Pengbo Bo and Michael Bartoň. On initialization of milling paths for 5 -axis flank $\mathrm{CNC}$ machining of free-form surfaces with general milling tools. Computer Aided Geometric Design, 71:30-42, 2019.

[11] Jinesh Machchhar, Denys Plakhotnik, and Gershon Elber. Precise algebraic-based swept volumes for arbitrary free-form shaped tools towards multi-axis CNC machining verification. Computer-Aided Design, 2017.

[12] Pengbo Bo, Michael Bartoň, Denys Plakhotnik, and Helmut Pottmann. Towards efficient 5-axis flank CNC machining of free-form surfaces via fitting envelopes of surfaces of revolution. Computer-Aided Design, 79:1$11,2016$.

[13] C. Li, S. Bedi, and S. Mann. Flank millable surface design with conical and barrel tools. Computer-Aided Design and Applications, 5:461-470, 2008.

[14] Faydor L Litvin and Alfonso Fuentes. Gear geometry and applied theory. Cambridge University Press, 2004.

[15] Hartmuth Müller and Joachim Thomas. Face-off: face hobbing vs. face milling. Gear Solutions, 5(54):49-60, 2007.

[16] Fritz Klocke, Markus Brumm, and Julian Staudt. Quality and surface of gears manufactured by free form milling with standard tools. In Proceedings of the International Gear Conference, Lyon, France, pages 26-28, 2014.

[17] Álvaro Álvarez, Amaia Calleja, Naiara Ortega, and Luis de Lacalle. Five- 
axis milling of large spiral bevel gears: toolpath definition, finishing, and shape errors. Metals, 8(5):353, 2018.

[18] Wuhao Zhuang, Lin Hua, Xinghui Han, and Fangyan Zheng. Design and hot forging manufacturing of non-circular spur bevel gear. International Journal of Mechanical Sciences, 133:129-146, 2017.

[19] Álvaro Álvarez, Amaia Calleja, Mikel Arizmendi, Haizea González, and Luis Lopez de Lacalle. Spiral bevel gears face roughness prediction produced by CNC end milling centers. Materials, 11(8):1301, 2018.

[20] Xiao-yu Yang and Jin-yuan Tang. Research on manufacturing method of CNC plunge milling for spur face-gear. Journal of Materials Processing Technology, 214(12):3013-3019, 2014.

[21] Fritz Klocke, Markus Brumm, and Julian Staudt. Quality and surface of gears manufactured by free form milling with standard tools. In Proceedings of the International Gear Conference, Lyon, France, pages 26-28, 2014.

[22] Włodzimierz Wilk and Jacek Tota. Modern technology of the turbine blades removal machining. In Proc. of the $* 8$ th Int. Conf.Advanced manufacturing Operations, Scientific reports, pages 347-355, 2008.

[23] Haizea González Barrio, Amaia Calleja Ochoa, Octavio Manuel Pereira Neto, Naiara Ortega Rodríguez, Luis Norberto López de Lacalle Marcaide, and Michael Barton. Super abrasive machining of integral rotary components using grinding flank tools. 2018.

[24] Pengbo Bo, Michael Bartoň, and Helmut Pottmann. Automatic fitting of conical envelopes to free-form surfaces for flank CNC machining. Computer-Aided Design, 91:84-94, 2017.

[25] DK Aspinwall, SL Soo, DT Curtis, and AL Mantle. Profiled superabrasive grinding wheels for the machining of a nickel based superalloy. CIRP annals, 56(1):335-338, 2007.

[26] DIN EN ISO 4288. Geometrical product specifications (gps)surface texture: Profile method: Rules and procedures for the assessment of surface texture. 1996.

[27] Surface finish ranges and tolerances. https://www. cnccookbook. com/surface-finish-chart-symbols-measure-calculators/ 


\section{Figures}

(a)
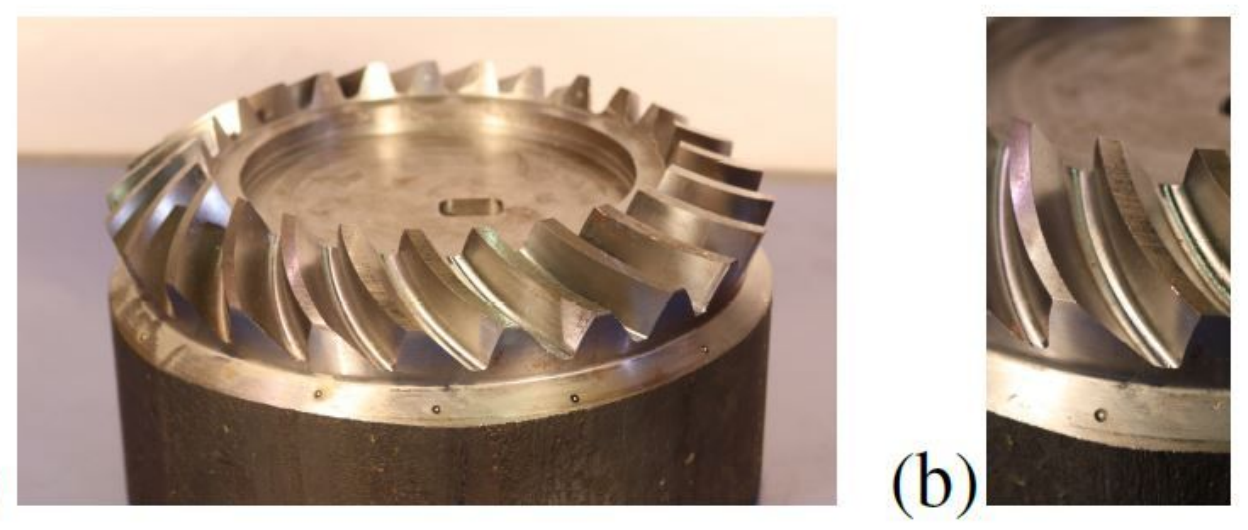

(c)

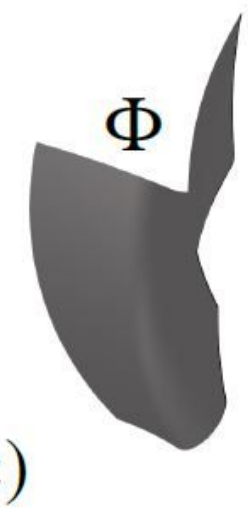

\section{Figure 1}

Spiral bevel gear. (a) A gear with curved teeth. (b) A zoom-in to one interdental cavity (aka "valley") between two teeth and its CAD model that is formed by a doubly curved free-form surface $\Phi$ (c).

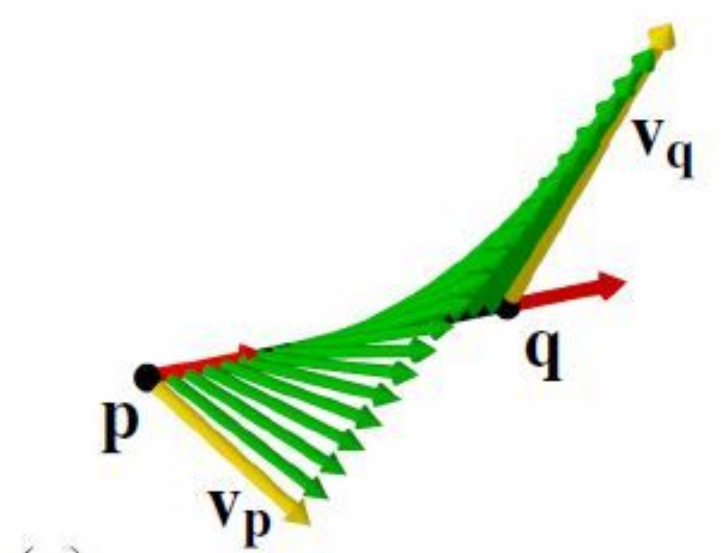

(a)

\section{Figure 2}

An instantaneous motion of the axis of the milling tool $\mathrm{pq}$ is determined by two velocity vectors $\mathrm{vp}$ and $\mathrm{vq}$ (yellow) that have to satisfy Eq. (??). This constraint is geometrically interpreted as the projections of vp and vq onto pq are equally oriented vectors of the same length (red). (b) A milling tool $\Psi$ is conceptualized as a one-parameter family of spheres (transparent) centered along pq and determined by $\mathrm{d}(\mathrm{s})$; here a hyperbolic tool is shown. 
(a)

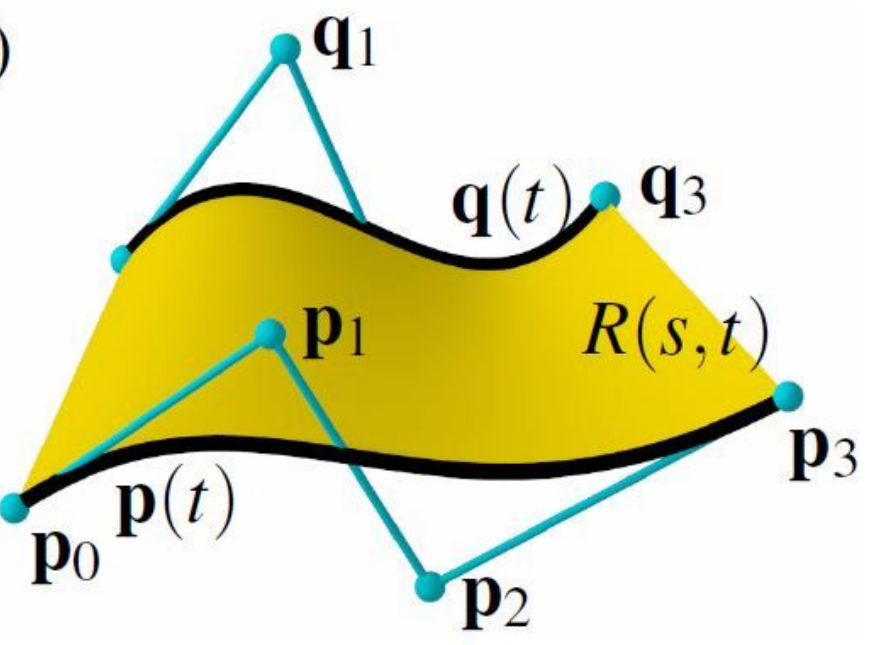

(b)

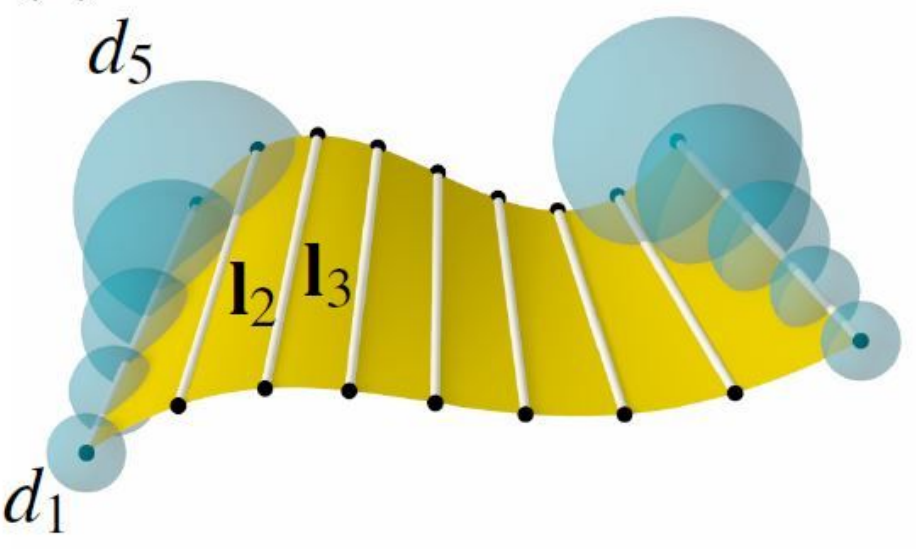

Figure 3

General milling tool and its motion. (a) A motion of the axis I of the milling tool is a ruled surface $\mathrm{R}(\mathrm{s}, \mathrm{t})$ that is controlled by two boundary curves $p(t)$ and $q(t)$. A single $(1 \times 3)$ tensor-product $B^{\prime}$ ezier patch is shown; the control points p0, .., q3 (blue) are the unknows for the optimization. (b) The general milling tool is considered as a one-parameter family of spheres centered along I. The vector of radii $d=(d 1, \ldots, d n)$ is also the unknown in our optimization framework and has to be the same for every position of the axis $11, \ldots, \operatorname{lm}$ (here $n=5, m=9$ ). 


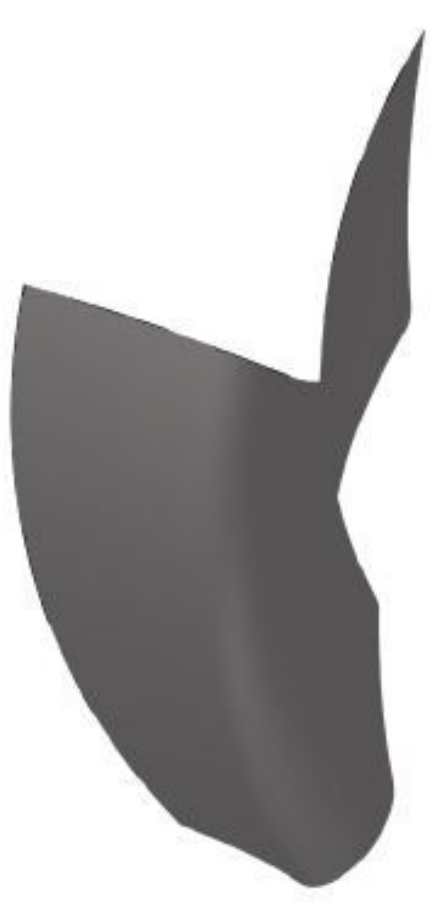

(a)

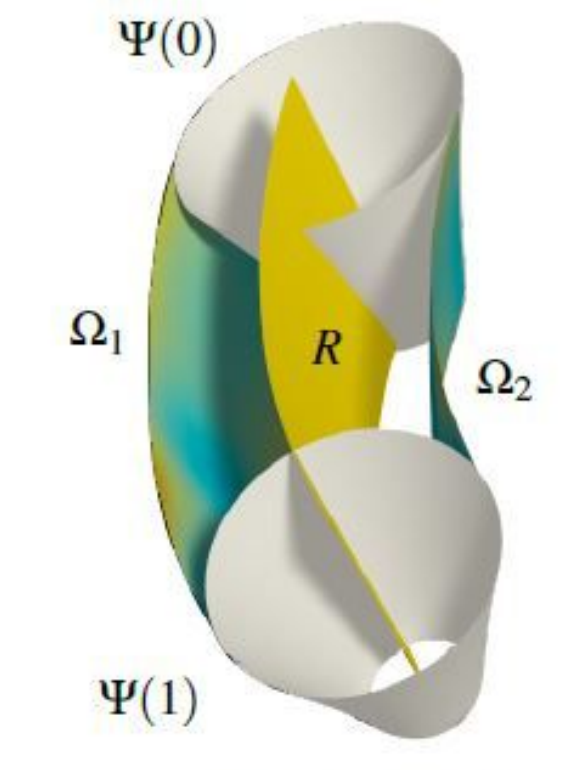

(c)

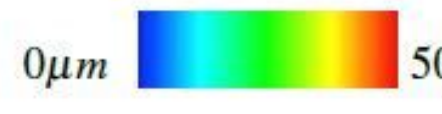

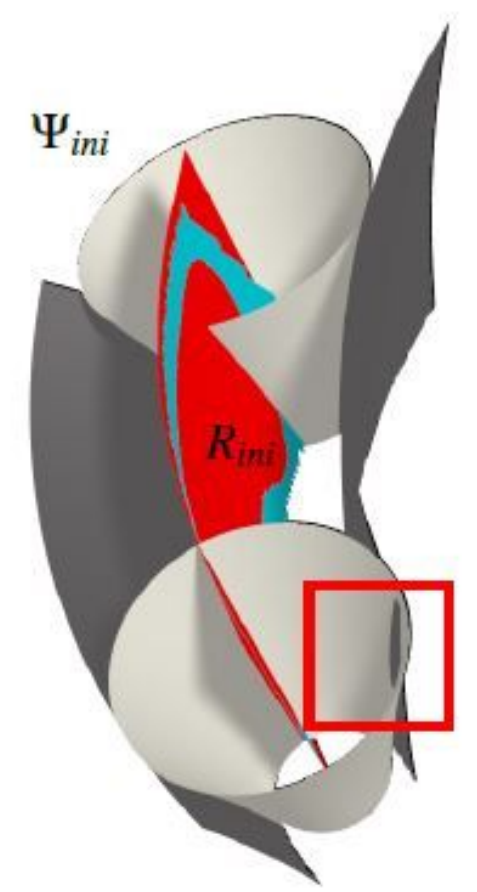

(b)

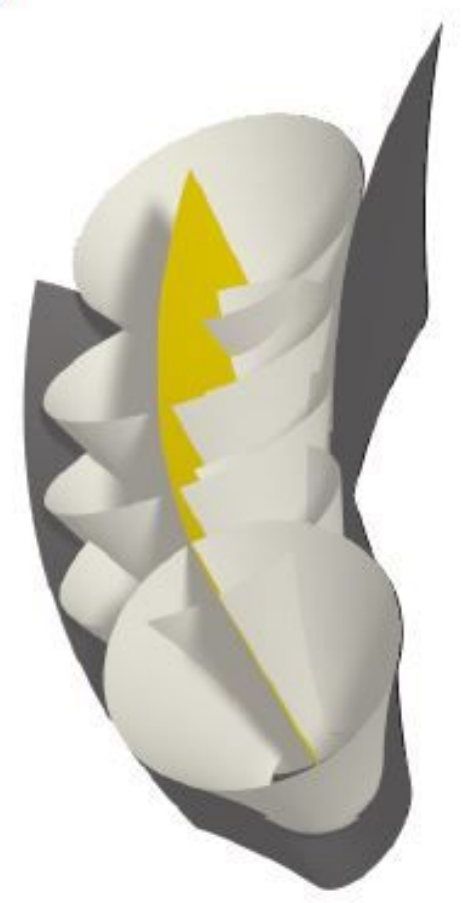

$50 \mu m$ (d)

\section{Figure 4}

Design of the custom-shaped tool. (a) A cavity bewteen two teeth represented as a spline surface. (b) The self-bisector B (blue) is fitted by a ruled surface Rini (red) and an initial shape, $\Psi_{i n i}$, of the tool is computed. The initial tool and its initial position may penetrate the reference surface (framed in red). (c) The tool $\Psi$ and its trajectory R both undergo global optimization to minimize the error of the left $(\Omega 1)$ and right $(\Omega 2)$ envelopes from $\Phi$. The envelopes are color-coded by the distance error $\operatorname{dist}(\Phi \mathrm{i}-\Omega \mathrm{i}), \mathrm{i}=1,2$, that 
meets fine machining tolerance of $50 \mu \mathrm{m}$. (d) The final double-flank motion of the custom-shaped tool $\Psi$ through the gear valley.

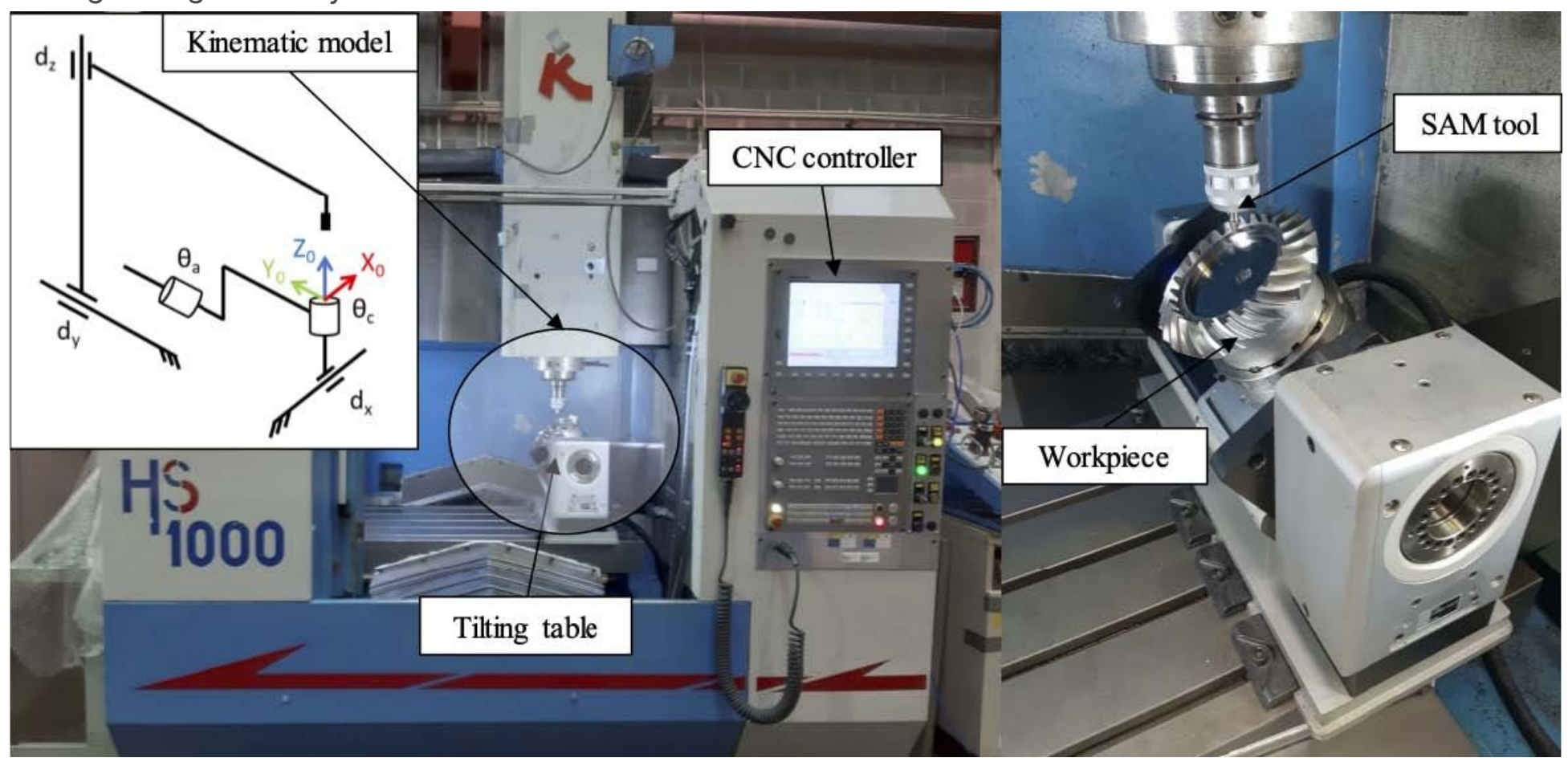

Figure 5

5-axis milling center. 


$\begin{array}{cc}\begin{array}{c}\text { Manufacturing } \\ \text { operations }\end{array} & \begin{array}{c}\text { Machining } \\ \text { time }\end{array}\end{array}$

0. Blank

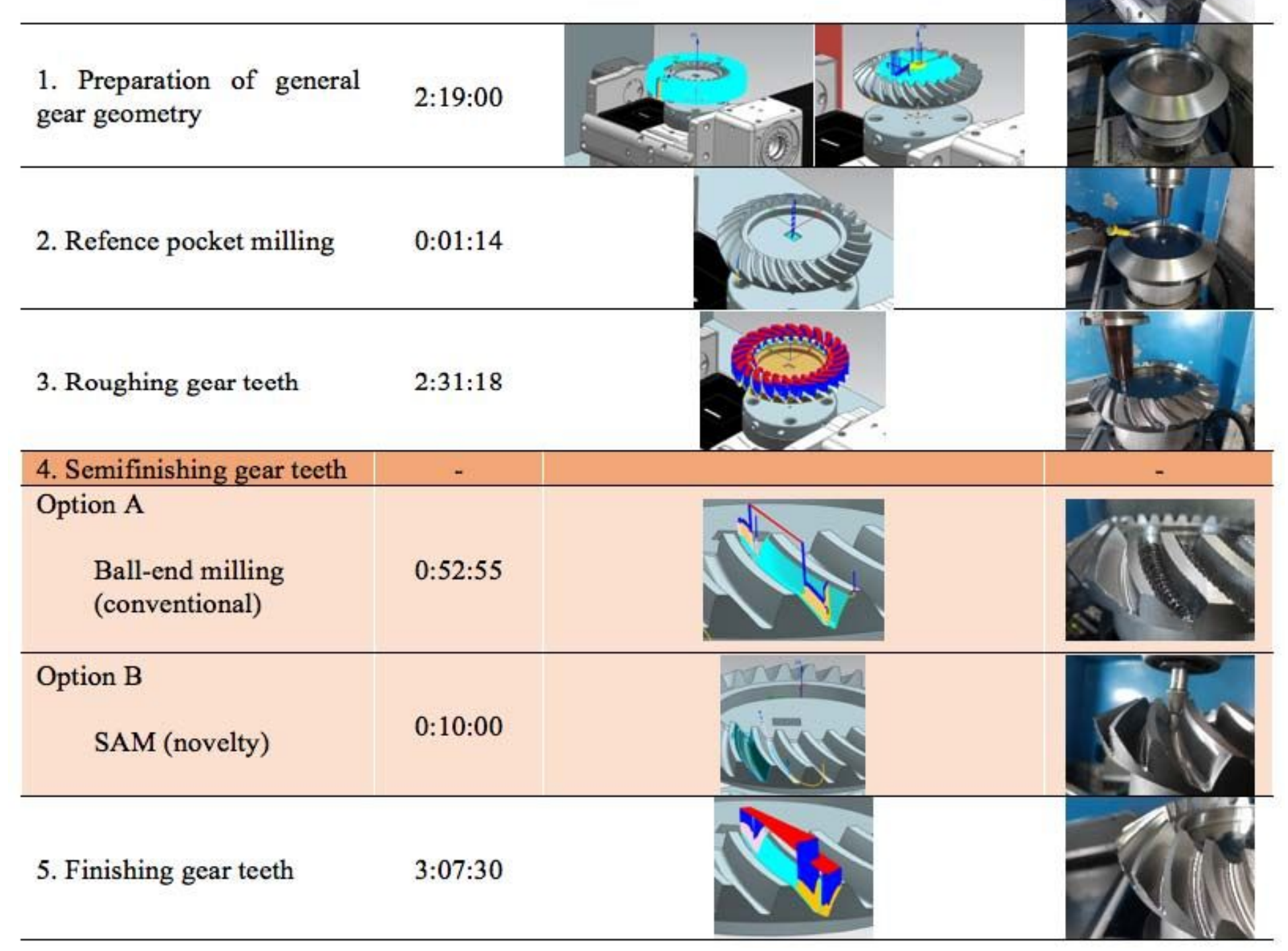

Figure 6

Progress and machining time summary of the whole gear manufacturing process.
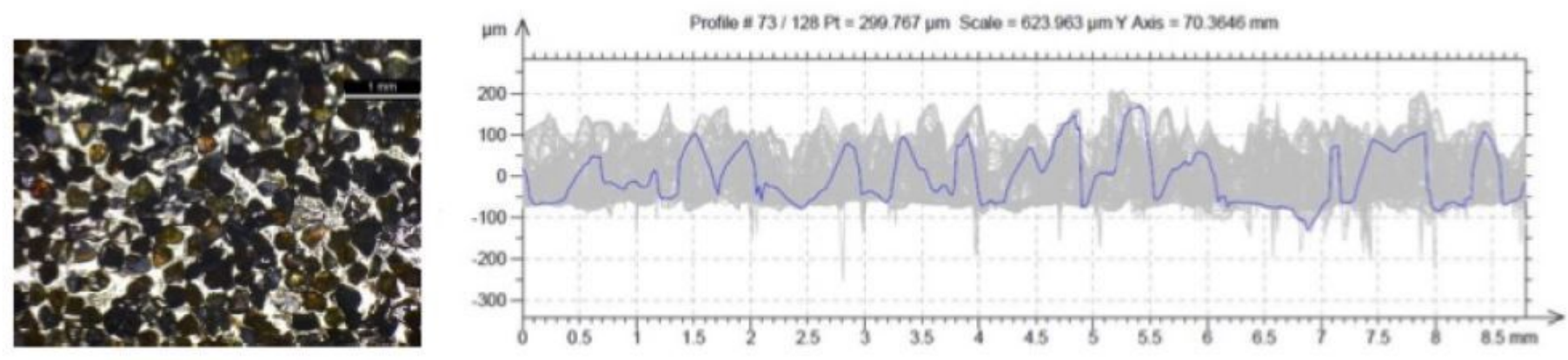
Figure 7

Grain distribution and profile.

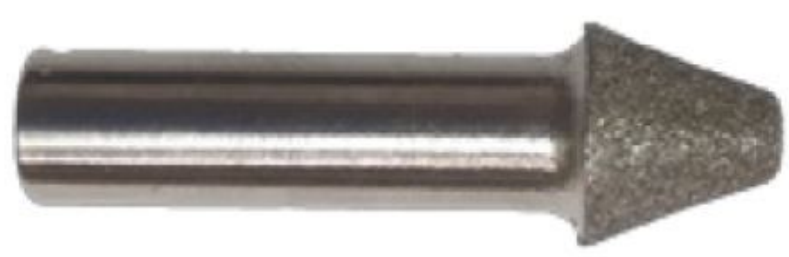

\section{Figure 8}

Custom-shaped grinding tool and its geometry.

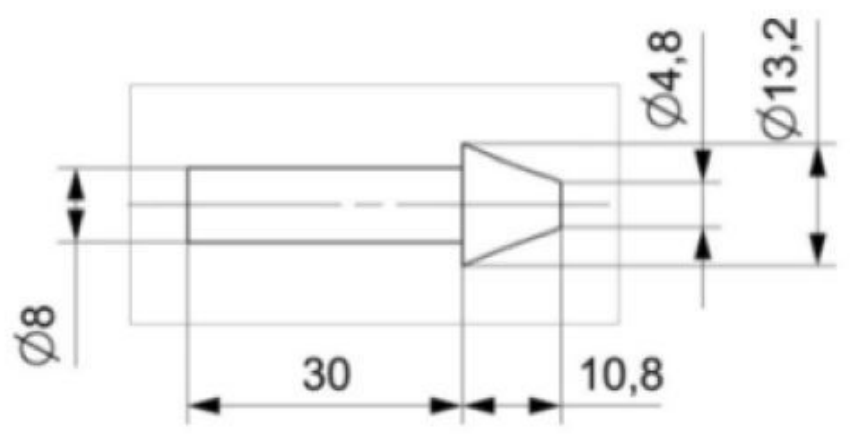




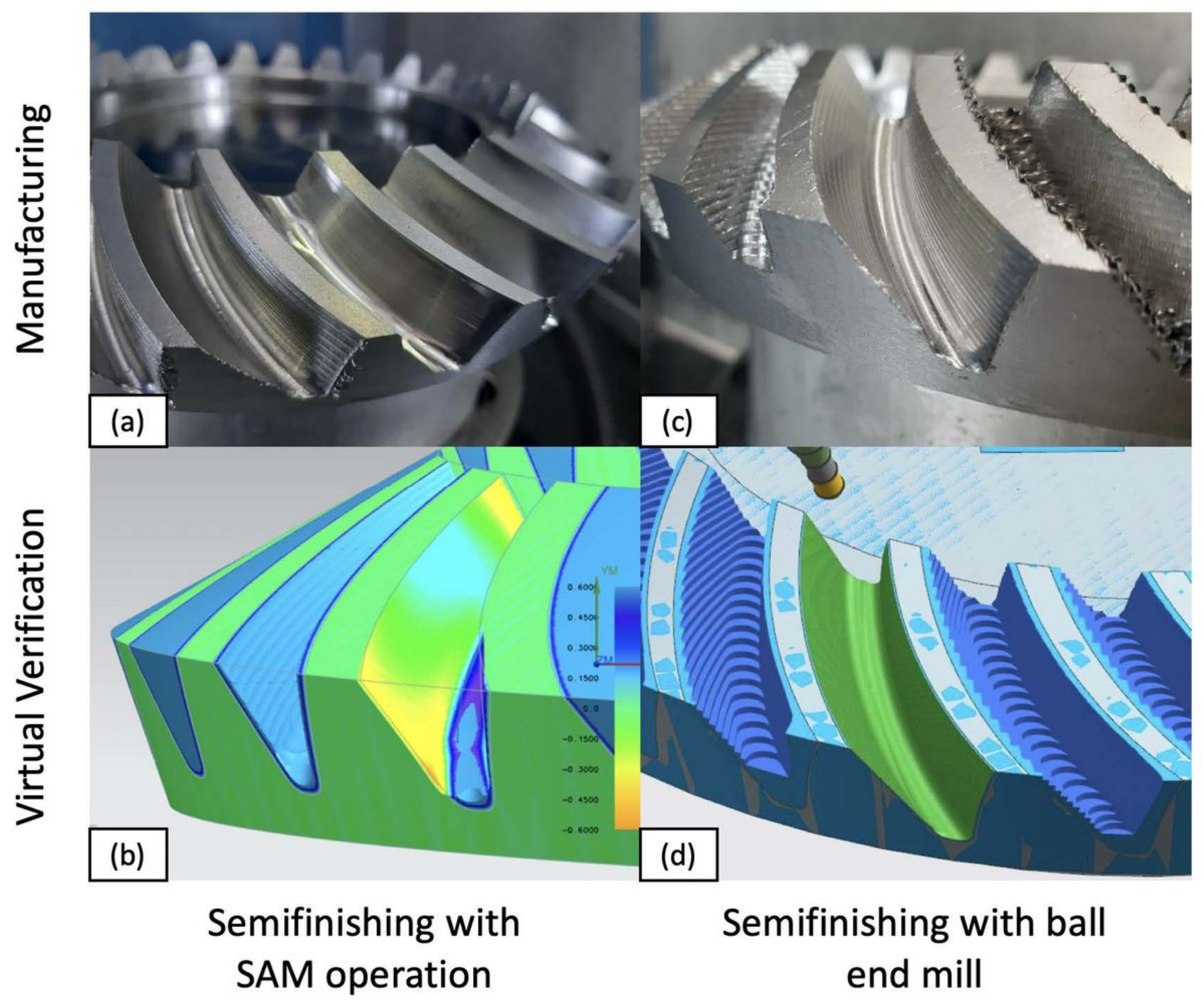

Figure 9

Spiral bevel semifinishing operation. (a) Manufacturing with SAM. (b) Virtual verification with SAM. (c) Manufacturing with ball end mill. (d) Virtual verification with ball end mill. 


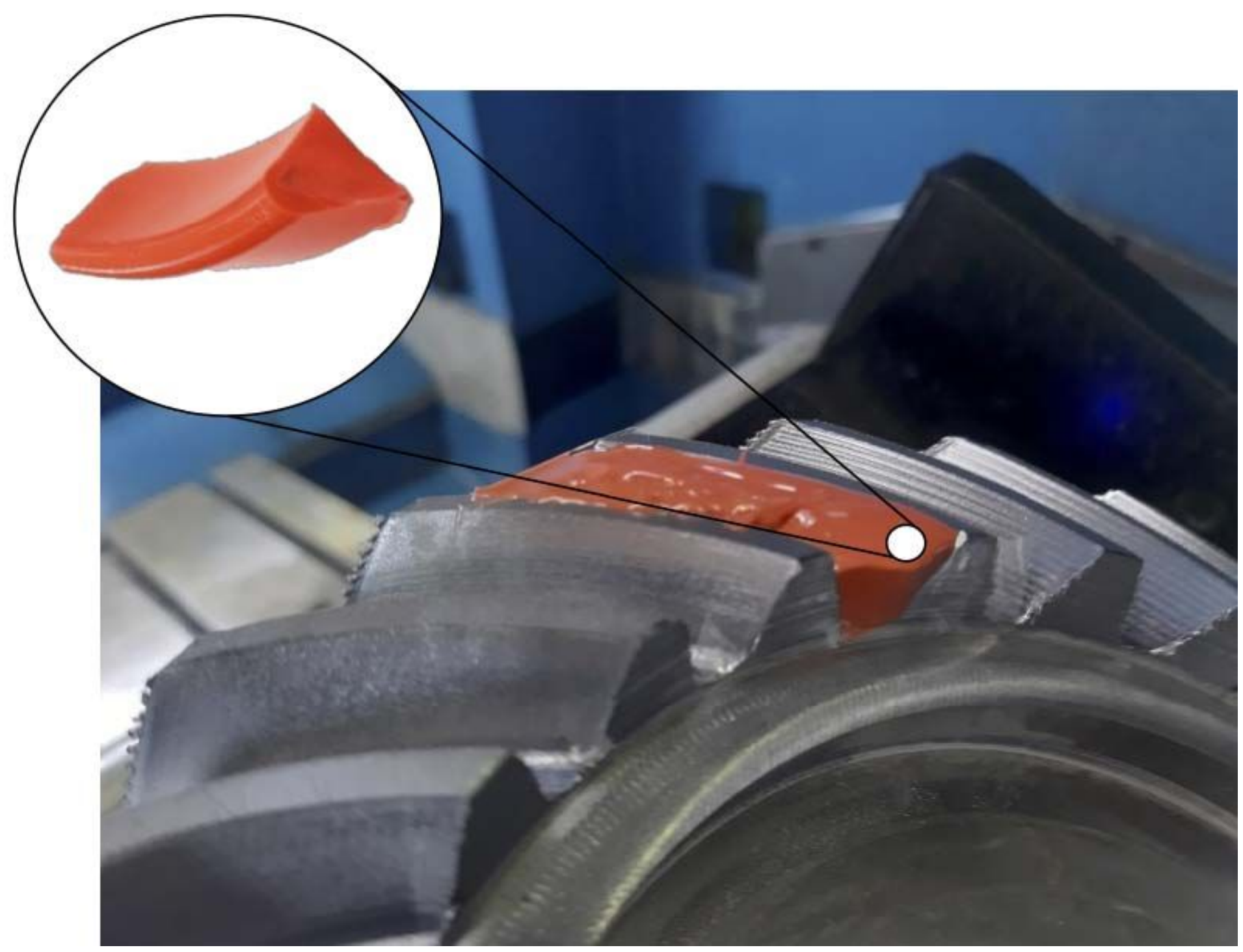

Figure 10

Curing process of the resin and its zoomed-in part after hardening. 


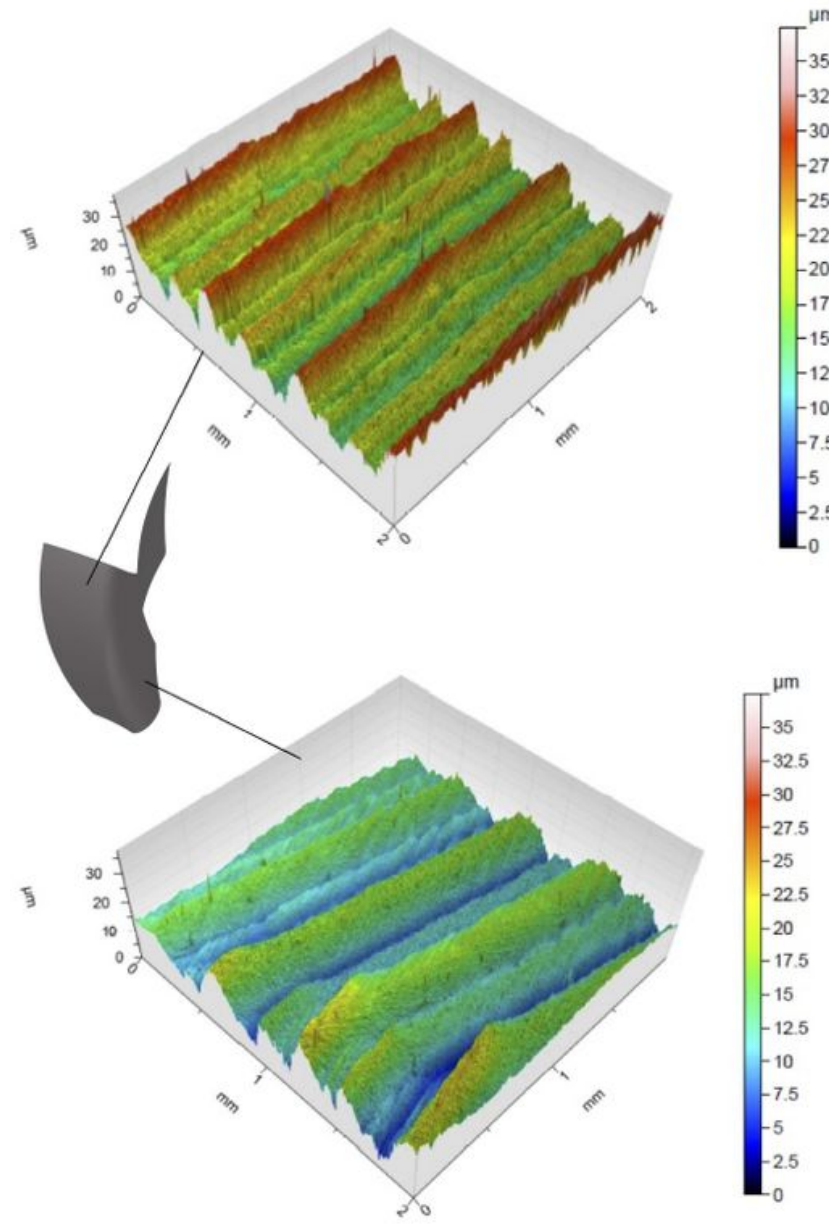

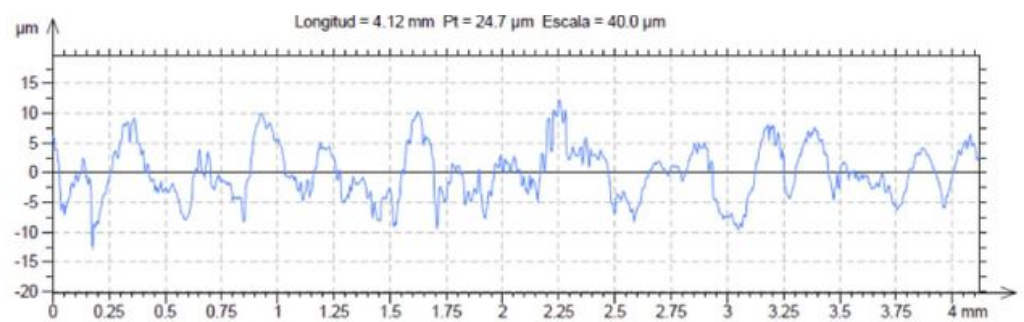

\begin{tabular}{|lll|}
\hline \multicolumn{3}{|l|}{ ISO 25178 } \\
\hline \multicolumn{3}{|l|}{ Heigth parameters } \\
\hline Sa & 3.87 & $\mu \mathrm{m}$ \\
\hline Sz & 37.1 & $\mu \mathrm{m}$ \\
\hline Sq & 4.78 & $\mu \mathrm{m}$ \\
\hline Sp & 17.5 & $\mu \mathrm{m}$ \\
\hline Sv & 19.6 & $\mu \mathrm{m}$ \\
\hline
\end{tabular}

\section{ISO 4287}

Amplitude parameters - Roughness profile

\begin{tabular}{lll|l}
$\mathbf{R a}$ & 3.23 & $\mu \mathrm{m}$ & Gaussian filter, $0.8 \mathrm{~mm}$
\end{tabular}

\begin{tabular}{lll|l}
$\mathbf{R z}$ & 17.2 & $\mu \mathrm{m}$ & Gaussian filter, $0.8 \mathrm{~mm}$
\end{tabular}

Surface parameters - Roughness profile

\begin{tabular}{lll|l}
$\mathbf{R S m}$ & 0.154 & $\mathrm{~mm}$ & Gaussian filter, $0.8 \mathrm{~mm}$
\end{tabular}

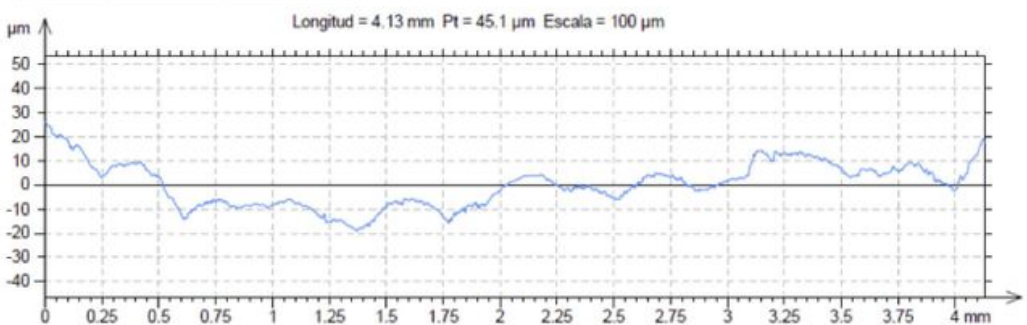

\begin{tabular}{|lll|}
\hline \multicolumn{3}{|l|}{ ISO 25178} \\
\hline \multicolumn{3}{|l|}{ Heigth parameters } \\
\hline Sa & 2.59 & $\mu \mathrm{m}$ \\
\hline Sz & 25.9 & $\mu \mathrm{m}$ \\
\hline Sq & 3.18 & $\mu \mathrm{m}$ \\
\hline Sp & 12.6 & $\mu \mathrm{m}$ \\
\hline Sv & 13.2 & $\mu \mathrm{m}$ \\
\hline
\end{tabular}

\section{ISO 4287}

Amplitude parameters - Roughness profile

\begin{tabular}{|ccc|c|}
\hline $\mathbf{R a}$ & 2.46 & $\mu \mathrm{m}$ & Gaussian filter, $0.8 \mathrm{~mm}$ \\
\hline $\mathbf{R z}$ & 11.8 & $\mu \mathrm{m}$ & Gaussian filter, $0.8 \mathrm{~mm}$ \\
\hline Surface & parameters & - Roughness profile \\
\hline RSm & 0.275 & $\mathrm{~mm}$ & Gaussian filter, $0.8 \mathrm{~mm}$ \\
\hline
\end{tabular}

\section{Figure 11}

Surface area and profile roughness parameters values of the left (top) and right (bottom) side of the gear tooth cavity. 